\title{
Spectroscopic evidence of large aspherical $\beta$-NAT particles involved in denitrification in the December 2011 Arctic stratosphere
}

\author{
Wolfgang Woiwode ${ }^{1}$, Michael Höpfner ${ }^{1}$, Lei Bi ${ }^{2, a}$, Michael C. Pitts ${ }^{3}$, Lamont R. Poole ${ }^{4}$, Hermann Oelhaf $^{1}$, \\ Sergej Molleker ${ }^{5}$, Stephan Borrmann ${ }^{5,6}$, Marcus Klingebiel ${ }^{6, b}$, Gennady Belyaev $^{7}$, Andreas Ebersoldt ${ }^{8}$, \\ Sabine Griessbach ${ }^{9}$, Jens-Uwe Grooß ${ }^{10}$, Thomas Gulde ${ }^{1}$, Martina Krämer ${ }^{10}$, Guido Maucher ${ }^{1}$, Christof Piesch $^{1}$, \\ Christian Rolf $^{10}$, Christian Sartorius ${ }^{1}$, Reinhold Spang ${ }^{10}$, and Johannes Orphal ${ }^{1}$ \\ ${ }^{1}$ Institute of Meteorology and Climate Research, Karlsruhe Institute of Technology, Karlsruhe, Germany \\ ${ }^{2}$ Department of Atmospheric Sciences, Texas A\&M University, College Station, TX 77843, USA \\ ${ }^{3}$ NASA Langley Research Center, Hampton, VA 23681, USA \\ ${ }^{4}$ Science Systems and Applications, Incorporated, Hampton, VA 23666, USA \\ ${ }^{5}$ Particle Chemistry Department, Max Planck Institute for Chemistry, Mainz, Germany \\ ${ }^{6}$ Institute for Physics of the Atmosphere (IPA), University of Mainz, Mainz, Germany \\ ${ }^{7}$ Myasishchev Design Bureau, Zhukovsky-5, Moscow Region, Russia \\ ${ }^{8}$ Institute for Data Processing and Electronics, Karlsruhe Institute of Technology, Karlsruhe, Germany \\ ${ }^{9}$ Jülich Supercomputing Centre (JSC), Forschungszentrum Jülich GmbH, 52425 Jülich, Germany \\ ${ }^{10}$ Institute of Energy and Climate Research (IEK-7), Forschungszentrum Jülich GmbH, 52425 Jülich, Germany \\ ${ }^{a}$ now at: School of Earth Sciences, Zhejiang University, Hangzhou, China, 310027 \\ bnow at: Atmosphere in the Earth System Department, Max Planck Institute for Meteorology, Hamburg, Germany
}

Correspondence to: Wolfgang Woiwode (wolfgang.woiwode@kit.edu)

Received: 16 February 2016 - Published in Atmos. Chem. Phys. Discuss.: 18 March 2016

Revised: 24 June 2016 - Accepted: 4 July 2016 - Published: 29 July 2016

\begin{abstract}
We analyze polar stratospheric cloud (PSC) signatures in airborne MIPAS-STR (Michelson Interferometer for Passive Atmospheric Sounding - STRatospheric aircraft) observations in the spectral regions from 725 to 990 and 1150 to $1350 \mathrm{~cm}^{-1}$ under conditions suitable for the existence of nitric acid trihydrate (NAT) above northern Scandinavia on 11 December 2011. The high-resolution infrared limb emission spectra of MIPAS-STR show a characteristic "shoulder-like" signature in the spectral region around $820 \mathrm{~cm}^{-1}$, which is attributed to the $\nu_{2}$ symmetric deformation mode of $\mathrm{NO}_{3}^{-}$in $\beta$-NAT. Using radiative transfer calculations involving Mie and T-Matrix methods, the spectral signatures of spherical and aspherical particles are simulated. The simulations are constrained using collocated in situ particle measurements. Simulations assuming highly aspherical spheroids with aspect ratios (AR) of 0.1 or 10.0 and a lognormal particle mode with a mode radius of $4.8 \mu \mathrm{m}$ reproduce the observed spectra to a high degree. A smaller lognormal mode with a mode radius of $2.0 \mu \mathrm{m}$, which is also
\end{abstract}

taken into account, plays only a minor role. Within the scenarios analyzed, the best overall agreement is found for elongated spheroids with $A R=0.1$. Simulations of spherical particles and spheroids with $\mathrm{AR}=0.5$ and 2.0 return results very similar to each other and do not allow us to reproduce the signature around $820 \mathrm{~cm}^{-1}$. The observed "shoulder-like" signature is explained by the combination of the absorption/emission and scattering characteristics of large highly aspherical $\beta$-NAT particles. The size distribution supported by our results corresponds to $\sim 9 \mathrm{ppbv}$ of gas-phase equivalent $\mathrm{HNO}_{3}$ at the flight altitude of $\sim 18.5 \mathrm{~km}$. The results are compared with the size distributions derived from the in situ observations, a corresponding Chemical Lagrangian Model of the Stratosphere (CLaMS) simulation, and excess gas-phase $\mathrm{HNO}_{3}$ observed in a nitrification layer directly below the observed PSC. The presented results suggest that large highly aspherical $\beta$-NAT particles involved in denitrification of the polar stratosphere can be identified by means of passive infrared limb emission measurements. 


\section{Introduction}

Nitric acid trihydrate (NAT) particles are known to be involved in denitrification and vertical downward transport of $\mathrm{HNO}_{3}$ in the Arctic and Antarctic winter stratosphere and thereby affect polar ozone chemistry (e.g., Waibel et al., 1999; Carslaw et al., 2002.; Grooß et al., 2014). $\beta$-NAT is the only nitric acid hydrate known to be thermodynamically stable in condensed state under the conditions of the polar winter stratosphere at temperatures below $\sim 195 \mathrm{~K}$ (Hanson and Mauersberger, 1988). The metastable modification $\alpha$-NAT is another potential PSC constituent at temperatures below $\sim 190 \mathrm{~K}$ and transforms irreversibly into $\beta$-NAT at higher temperatures (Tizek et al., 2004, and references therein). The presence of NAT in Arctic PSCs was confirmed by Voigt et al. (2000) using balloon-borne in situ measurements of PSC particles. Large $\mathrm{HNO}_{3}$-containing particles in Arctic PSCs likely consisting of NAT were also reported by Fahey et al. (2001).

Spang and Remedios (2003) and Spang et al. (2005) identified a "peak-like" signature in the spectral region around $820 \mathrm{~cm}^{-1}$ in spaceborne infrared PSC observations by CRISTA (Cryogenic Infrared Spectrometers and Telescopes for the Atmosphere) aboard the space shuttle and MIPAS (Michelson Interferometer for Passive Atmospheric Sounding) aboard Envisat. Based on Mie calculations, they suggested that the signature originated from the $\nu_{2}$ band of $\mathrm{NO}_{3}^{-}$in NAT particles with radii smaller than $\sim 2-3 \mu \mathrm{m}$. Höpfner et al. (2006a) attributed the "peak-like" signature around $820 \mathrm{~cm}^{-1}$ in the MIPAS-Envisat PSC observations unambiguously to $\beta$-NAT by using a new set of refractive indices of $\beta$-NAT (Biermann, 1998) and explicit radiative transfer simulations for particles in limb-sounding observations (Höpfner et al., 2002). Particle radii lower than $\sim 3 \mu \mathrm{m}$ and volume densities higher than $\sim 0.3 \mu \mathrm{m}^{3} \mathrm{~cm}^{-3}$ were derived. To our best knowledge, $\alpha$-NAT has not been identified in infrared field observations so far.

Further in situ observations of large potential NAT particles are reported by Brooks et al. (2003) and Molleker et al. (2014) and suggest extremely large particle sizes of $20 \mu \mathrm{m}$ and more. Particle backward trajectories associated with in situ observations of large potential NAT particles on 25 January 2010 suggest that the particles had experienced short growth times (Woiwode et al., 2014). However, the sizes of the largest particles derived from the in situ observations are not reproduced by a state-of-the-art chemistry transport model parameterization (Hoyle et al., 2013; Grooß et al., 2014).

The metastable nitric acid dihydrate (NAD) is another constituent, which is likely present in PSCs and might be involved in the formation of NAT (Worsnop et al., 1993). Nucleation experiments in the AIDA (Aerosol, Interactions, and Dynamics in the Atmosphere) cloud chamber under stratospheric conditions resulted in formation of NAD, while no NAT formation was observed (Stetzer et al., 2006). While there is little evidence of nitric acid hydrates other than NAT in the stratosphere, Kim et al. (2006) reported observations of $\beta$-NAT along with NAD as a minor component in the Antarctic stratosphere by the ILAS-II instrument. However, a systematic search in MIPAS-Envisat spectra measured during the Antarctic winter 2003 showed no indication of any NAD particles with radii equal to or less than about $1 \mu \mathrm{m}$ (Höpfner et al., 2006a). As discussed by Grothe et al. (2004), the spectroscopic data of NAD used in these studies closely correspond to the $\alpha$-NAD modification. Furthermore, another metastable high-temperature modification $\beta$-NAD has been identified by Grothe et al. (2004) in laboratory experiments at temperatures above $\sim 200 \mathrm{~K}$, which decomposes into $\beta$-NAT and NAM (nitric acid monohydrate) at considerably higher temperatures.

We use high-resolution MIPAS-STR spectra and collocated in situ observations during the high-altitude aircraft M-55 Geophysica (Stefanutti et al., 1999) ESSenCe (ESA Sounder Campaign 2011, Kaufmann et al., 2013) flight on 11 December 2011 inside an optically thin PSC to study the mid-infrared optical characteristics of large $\beta$-NAT particles in detail. The observations are brought into a synoptic context by comparisons with collocated observations by CALIPSO (Cloud-Aerosol Lidar and Infrared Pathfinder Satellite Observation, Pitts et al., 2011, 2013; Lambert et al., 2012, and references therein) and MIPAS-Envisat (Spang et al., 2005; Höpfner et al., 2006a, b, and references therein).

Using radiative transfer simulations, we model the PSC signatures in the MIPAS-STR observations and constrain the simulations with collocated in situ particle measurements. To simulate the signatures of spherical to highly aspherical $\beta$-NAT particles, we utilize the Mie code by Höpfner et al. (2002, 2006a) and the T-Matrix codes by Mishchenko and Travis (1998) and Bi et al. (2013). The assumption of highly aspherical $\beta$-NAT particles is supported by the work of Grothe et al. (2006), who synthesized highly aspherical $\beta$-NAT particles under laboratory conditions and obtained highly aspherical particles with different morphologies depending on the growth conditions.

In Sect. 2, we provide an overview of the meteorological situation, the MIPAS-STR and in situ PSC observations during the Geophysica flight, and collocated CALIPSO and MIPAS-Envisat observations. In Sect. 3, we analyze the PSC signatures in the MIPAS-STR spectra using radiative transfer simulations. In Sect. 4, we compare the particle size distribution supported by the radiative transfer simulations with the size distributions derived from the in situ observations, a Chemical Lagrangian Model of the Stratosphere (CLaMS) simulation, and excess $\mathrm{HNO}_{3}$ observed in a nitrification layer below the observed PSC. The results are summarized in Sect. 5 . 


\section{PSC observations on 11 December 2011}

\subsection{Instrumentation}

MIPAS-STR is a cryogenic Fourier transform spectrometer and provides high-resolution limb emission spectra in the mid-infrared (Piesch et al., 1996). Instrument characteristics, data processing, validation, and PSC observations by MIPAS-STR are discussed by Woiwode et al. (2012, 2014, 2015). Here, we use MIPAS-STR channel 1 (725 to $\left.990 \mathrm{~cm}^{-1}\right)$ and channel $2\left(1150\right.$ to $\left.1350 \mathrm{~cm}^{-1}\right)$ spectra recorded at a spectral sampling of $0.036 \mathrm{~cm}^{-1}$ and with an apodized spectral resolution of $0.069 \mathrm{~cm}^{-1}$ for cloud detection, retrievals of temperature and trace gases, and the analysis of PSC signatures. The apodized noise equivalent spectral radiance of the MIPAS-STR spectra is typically $\sim 10 \times 10^{-9} \mathrm{~W} \mathrm{~cm}^{-2} \mathrm{sr}^{-1} \mathrm{~cm}$ in channel 1 and $\sim 8 \times$ $10^{-9} \mathrm{~W} \mathrm{~cm}^{-2} \mathrm{sr}^{-1} \mathrm{~cm}$ in channel 2 . The vertical spacing of the utilized MIPAS-STR limb observations is mostly $1.0 \mathrm{~km}$ ( $1.5 \mathrm{~km}$ for the lowest limb views) between $\sim 5 \mathrm{~km}$ and flight altitude. Additional upward sampling provides limited information on the atmospheric scenario above (mainly column information). The horizontal along-track sampling during the discussed flight is $\sim 33 \mathrm{~km}$.

In situ particle observations aboard the Geophysica were performed by the Forward Scattering Spectrometer Probe 100 (FSSP-100) and the Cloud Droplet Probe (CDP) (Molleker et al., 2014, and references therein). Both instruments detect forward scattering of laser light at $633 \mathrm{~nm}$ (FSSP-100) and $658 \mathrm{~nm}$ (CDP). From the observations, particle size distributions in the ranges from 1.05 to $37 \mu \mathrm{m}$ (FSSP$100)$ and 4 to $50 \mu \mathrm{m}$ (CDP) are derived. The FSSP-100 and CDP measurements discussed here were evaluated using Mie theory, and particle sizes are indicated in diameter.

CALIOP (Cloud-Aerosol LIdar with Orthogonal Polarization) aboard the CALIPSO satellite is a two-wavelength polarization-sensitive lidar. CALIOP total and perpendicular backscatter coefficient observations at $532 \mathrm{~nm}$ are used for PSC identification and classification according to Pitts et al. (2011, 2013). CALIPSO is part of the A-train constellation and provides observations up to $82^{\circ}$ latitude in each hemisphere. The vertical and horizontal resolutions of the PSC product are $180 \mathrm{~m}$ and $5 \mathrm{~km}$, respectively.

MIPAS-Envisat is the spaceborne version of the MIPAS instruments (Fischer et al., 2008). Here, we use the spectral window from 780 to $860 \mathrm{~cm}^{-1}$ in channel A. The shown observations were performed in the reduced-resolution nominal mode and have an apodized spectral resolution of $0.121 \mathrm{~cm}^{-1}$ (von Clarmann et al., 2009). The apodized noise equivalent spectral radiance of the MIPAS-Envisat channel A spectra is typically $11-19 \times 10^{-9} \mathrm{~W} \mathrm{~cm}^{-2} \mathrm{sr}^{-1} \mathrm{~cm}$. Envisat was deployed on a polar orbit, with the MIPAS-Envisat observations reaching up to $\sim 89^{\circ} \mathrm{N}$. The vertical and horizontal samplings of the observations discussed here were $\sim 1.5 \mathrm{~km}$ and $\sim 3.5^{\circ}$ in latitude $(\sim 390 \mathrm{~km})$, respectively.

\subsection{Meteorological conditions, flight overview, and PSC observations aboard the Geophysica}

Figure 1 gives an overview of the meteorological situation and the observations from the Geophysica on 11 December 2011. The flight was performed between 11:05 and 15:00 UTC, with takeoff and landing in Kiruna, Sweden. Also shown are the potential vorticity contours from the ECMWF (European Centre for Medium-Range Weather Forecasts) ERA-Interim reanalysis. The contours indicate that most of the flight was performed inside the polar vortex according to the criterion of Nash et al. (1996). The $430 \mathrm{~K}$ potential temperature level corresponds to a geometric altitude of $\sim 18 \mathrm{~km}$, which was the approximate flight altitude of the Geophysica. The flight was performed in a clockwise pattern, and the polar vortex edge was crossed twice during flight legs $\mathrm{b}$ and $\mathrm{c}$.

Open black circles in Fig. 1 indicate the tangent points of the MIPAS-STR limb observations. The horizontal distance of the tangent points from the flight path increases from a few tens of kilometers to $\sim 400 \mathrm{~km}$ with decreasing tangent altitude. The cloud index method by Spang et al. (2004) identifies cloud-affected MIPAS observations. In the case of MIPAS-Envisat, cloud index values higher than 4 are considered cloud-free, and values between 4 and 1 indicate the transition from slightly cloud-affected to opaque conditions. In Fig. 1, MIPAS-STR observations with tangent altitudes $>16 \mathrm{~km}$ (i.e., close to the flight track) and cloud index values $<3$ are marked by filled circles. Here, we use a less conservative cloud index threshold of 3 to locate the PSC around the flight track north of $\sim 68^{\circ} \mathrm{N}$ sharply. The flight section where the FSSP-100 and CDP detected the PSC (see Molleker et al., 2014) is indicated by the red line and coincides well with the MIPAS-STR PSC observations.

Figure 2 shows the vertical flight profile together with the MIPAS-STR tangent points color-coded with the cloud index. After ascent, the Geophysica performed flight legs a and $\mathrm{b}$ at an approximately constant flight altitude of $\sim 17 \mathrm{~km}$. In leg c, an approximately constant flight altitude of $\sim 18 \mathrm{~km}$ was maintained until $\sim 13: 30$ UTC. Finally, the Geophysica climbed to the ceiling altitude of $\sim 18.5 \mathrm{~km}$, until the descent phase was entered at $\sim 14: 20$ UTC in leg e. From $\sim 13: 30$ to 14:20 UTC, low cloud index values indicate the presence of a PSC around flight altitude. Particularly low cloud index values are found in the time interval where the in situ instruments detected the PSC.

Several of the MIPAS-STR limb scans during the PSC encounter show low cloud index values from flight altitude towards lower stratospheric altitudes. However, no sharp lower boundary of the PSC can be assigned, since the lower limb views are already affected by the PSC around flight altitude. At tropospheric altitudes, the cloud index values decrease towards 1 between 4 and $10 \mathrm{~km}$ and indicate tropospheric clouds. 


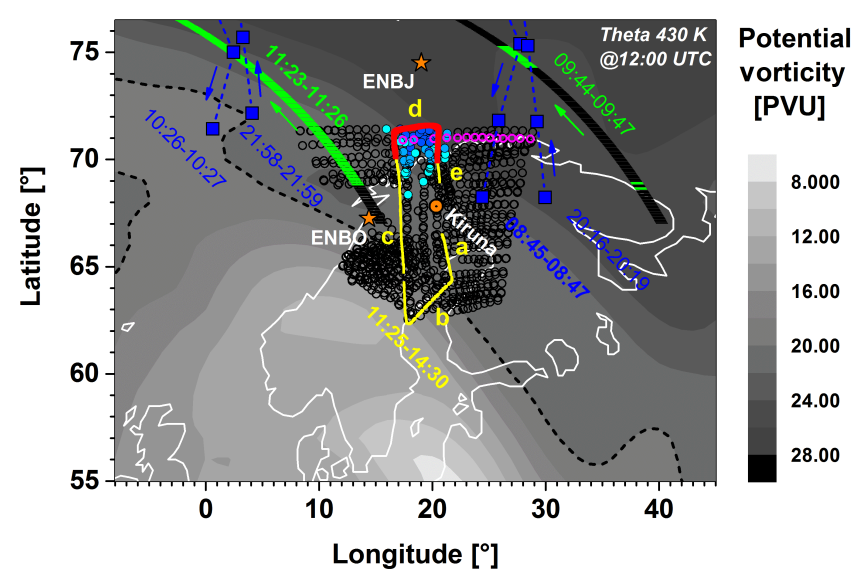

Figure 1. Flight path (yellow line) of the Geophysica and meteorological situation during the PSC flight on 11 December 2011. Grey shading: ECMWF ERA-Interim potential vorticity at the potential temperature level of $430 \mathrm{~K}$. Black dashed line: polar vortex edge according to Nash et al. (1996). Open black circles: MIPAS-STR tangent points. Filled circles (cyan to blue): MIPAS-STR tangent points $>16 \mathrm{~km}$ with cloud index $<3$. Magenta circles: MIPAS-STR limb scan associated with spectra considered in radiative transfer simulations. Red line: flight section of FSSP-100 and CDP PSC observations. Horizontal green/black bars: CALIPSO PSC observations (green: PSC detected at $21 \mathrm{~km}$ ). Filled blue squares: MIPASEnvisat observations at 18.4 to $19.0 \mathrm{~km}$ exhibiting PSC signatures. Orange stars: radiosonde launch sites Bodø (ENBO) and Bjornoya (ENBJ).

Molleker et al. (2014) calculated backward trajectories for large particles sampled during the discussed flight and found temperatures close to the frost point $\sim 20 \mathrm{~h}$ before the flight. While the model temperatures were too warm for ice nucleation, ice particles might have nucleated during lee-waveinduced cooling above Greenland not resolved by the model. Therefore, it is unclear whether the observed particles have nucleated heterogeneously from ice and/or according to a different mechanism.

\subsection{CALIPSO PSC observations}

Figure 3 shows the results of the closest-matching CALIPSO observations (see Fig. 1, 11:23 to 11:26 UTC). The observations were performed $\sim 2 \mathrm{~h}$ prior to the Geophysica PSC encounter. The scattering ratio at $532 \mathrm{~nm}$ is the ratio of total volume backscatter to molecular backscatter and is sensitive to all types of cloud particles (Fig. 3a). The perpendicular backscatter coefficient at $532 \mathrm{~nm}$ is sensitive to depolarization of the backscattered light by aspherical cloud particles (Fig. 3b). The combination of the inverse scattering ratio and the perpendicular backscatter at $532 \mathrm{~nm}$ is utilized for the CALIPSO PSC detection and classification according to Pitts et al. (2011, 2013) (Fig. 3c). The method classifies mixtures dominated by STS (STS mix), mixtures of NAT and STS with increasing number densities of NAT (Mix1, Mix2,

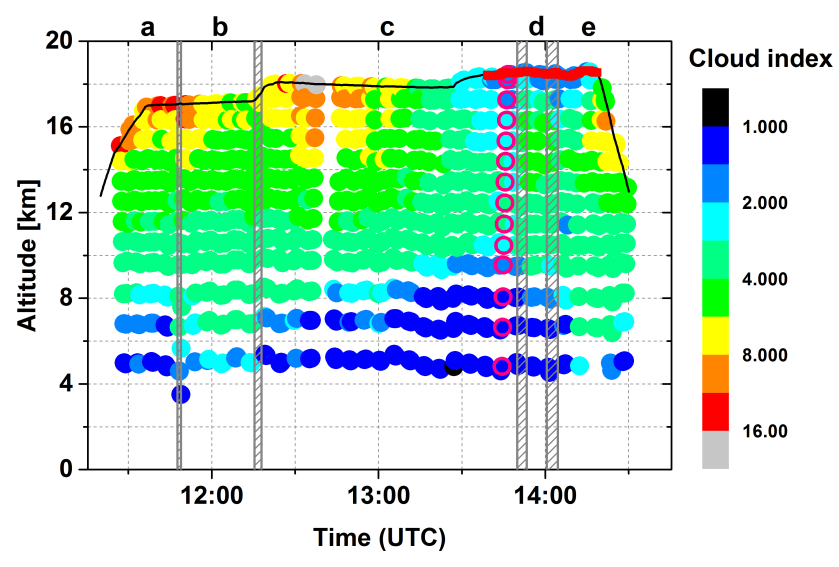

Figure 2. Vertical flight profile of the Geophysica (black line) and vertical distribution of the MIPAS-STR tangent points, color-coded with the cloud index (filled circles). Open magenta circles: MIPASSTR limb scan associated with spectra considered in radiative transfer simulations. Red line: flight section of FSSP-100 and CDP PSC observations. Grey hatched areas: turns between different legs.

and Mix2-enh), ice-containing mixtures (Ice mix), and wave ice (Wave ice). Thereby, the utilization of the perpendicular backscatter at $532 \mathrm{~nm}$ enhances the detection of tenuous PSCs containing low number densities of NAT particles.

Usually, only CALIPSO nighttime observations are used for PSC detection and classification. Daytime CALIPSO observations are characterized by strongly enhanced noise, making it much more difficult to detect enhanced backscatter from tenuous PSCs. In the case being discussed, strict exclusion of daytime observations would mean excluding CALIPSO observations south of $72.15^{\circ} \mathrm{N}$ and would greatly decrease the number of close-matching observations. To increase the number of close-matching CALIPSO observations, we included twilight observations down to $67^{\circ} \mathrm{N}$. The comparison of the nighttime section $>72.15^{\circ} \mathrm{N}$ and the twilight sections in the cross sections in Fig. 3 however provides a consistent picture of an extended PSC stretching from $\sim 69^{\circ} \mathrm{N}$ northwards. False positive signal enhancements due to noise during twilight can be clearly distinguished as patchy spots at latitudes south of $\sim 69^{\circ} \mathrm{N}$.

In the vicinity of the Geophysica PSC observations, the scattering ratio and the perpendicular backscatter (Fig. 3a and b) faintly indicate a PSC between $\sim 19$ and $23 \mathrm{~km}$. Between 69.0 and $71.4^{\circ} \mathrm{N}$, the cluster of CALIPSO data with low scattering ratios and perpendicular backscatter values slightly exceeding the noise level suggests PSC layers consisting of NAT-containing mixtures (Fig. 3c). Much stronger signal enhancements and a more diverse composition are evident in the part of the PSC extending northward, which spans altitudes from $\sim 18$ to $25 \mathrm{~km}$. 
(a)

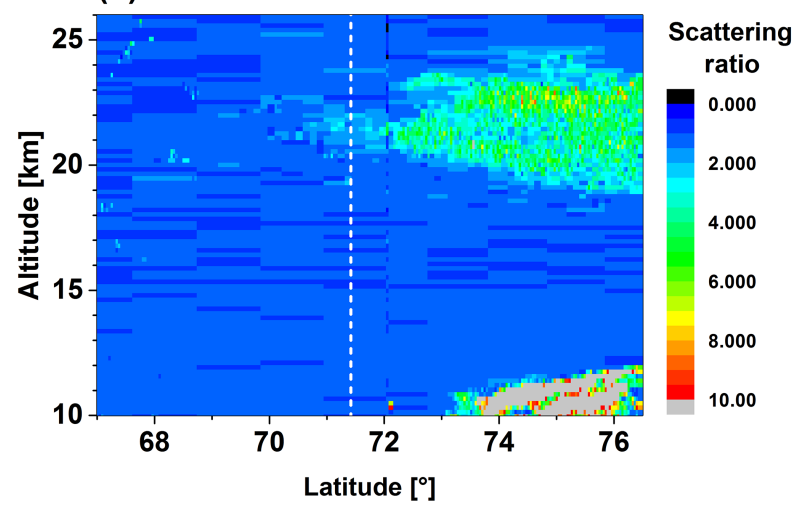

(b)

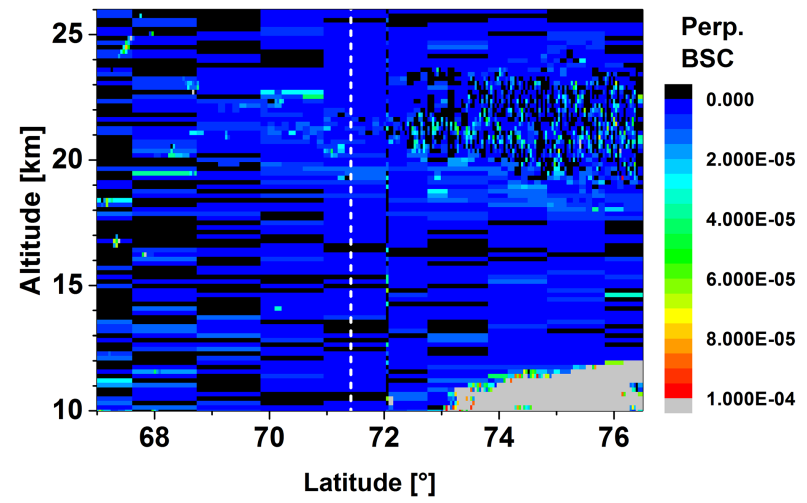

(c)

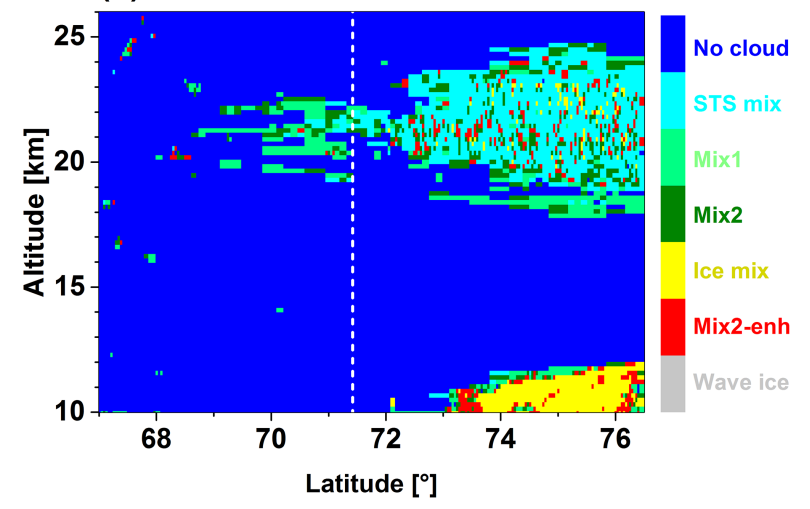

Figure 3. CALIPSO PSC observations associated with the Geophysica flight on 11 December 2011. (a) Scattering ratio at $532 \mathrm{~nm}$. (b) Perpendicular backscatter (BSC) at $532 \mathrm{~nm}$. (c) PSC classification according to Pitts et al. (2011, 2013). White dashed lines: latitude of northernmost observations aboard the Geophysica.

\subsection{PSC signatures in airborne and spaceborne MIPAS observations}

Figure 4a and $\mathrm{b}$ show the MIPAS-STR channel 1 and 2 spectra associated with the uppermost limb views of the limb scans during the time interval where the in situ instruments detected the PSC (13:39 to 14:19 UTC). Within limb scans at the bottom of a PSC, the views in approximately hori-
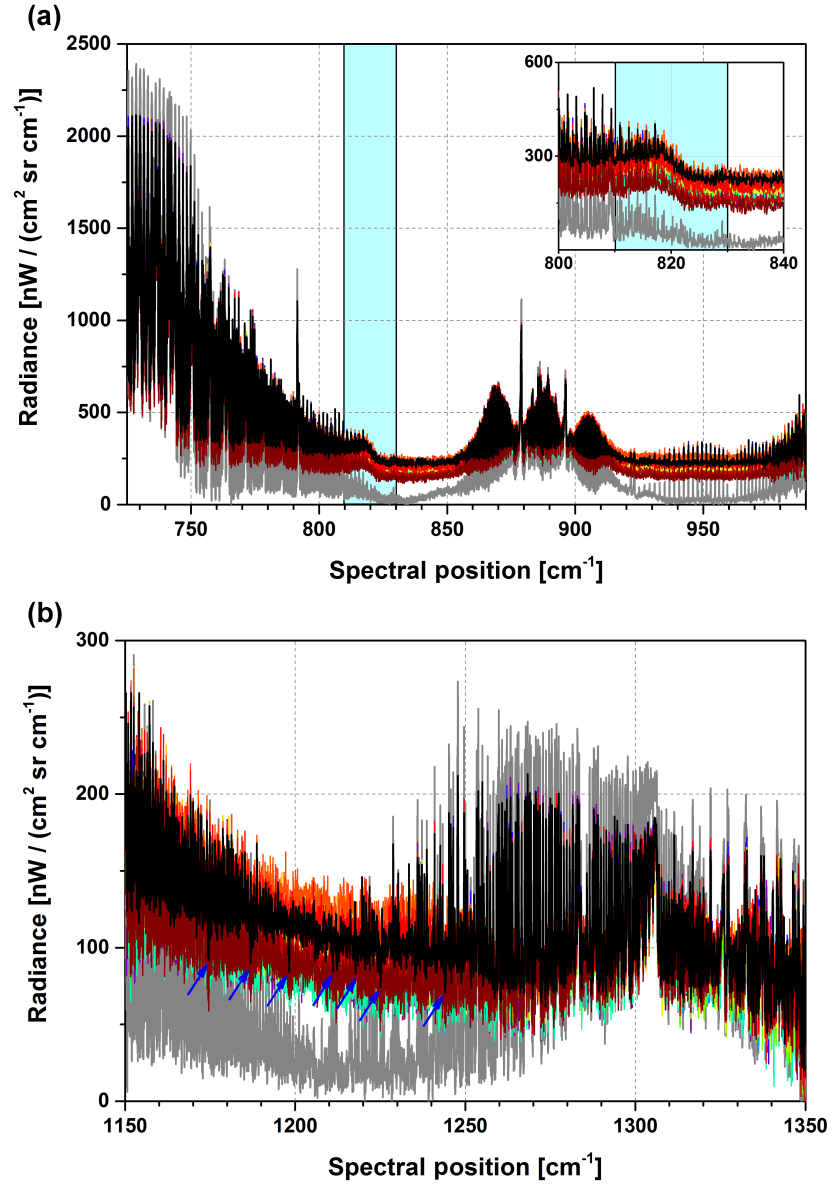

Figure 4. (a) MIPAS-STR channel 1 spectra of approximately horizontal limb views. Colored spectra: observations inside PSC in the time interval of the in situ PSC observations. Black spectrum: PSC observation analyzed in Sect. 3 (elevation angle $-0.25^{\circ}$ vs. horizon, flight altitude $18.507 \mathrm{~km}$, and tangent altitude $18.445 \mathrm{~km}$ ). Grey spectrum: corresponding observation under cloud-free conditions. Spectral region of a "shoulder-like" signature around $820 \mathrm{~cm}^{-1}$ attributed to $\beta$-NAT particles shaded in cyan. (b) Same as panel (a) but for MIPAS-STR channel 2. Blue arrows denote prominent $\mathrm{H}_{2} \mathrm{O}_{(\mathrm{g})}$ absorption signatures from scattering of tropospheric radiation by PSC particles.

zontal geometry typically exhibit the strongest PSC signals. The observation at 13:47 UTC (black) belongs to the limb scan marked in magenta in Figs. 1 and 2. A cloud-free observation at 12:27 UTC (grey) is shown for comparison. Compared to the cloud-free observation, the PSC spectra show a strong continuum-like offset of typically 150 to $300 \times$ $10^{-9} \mathrm{~W} \mathrm{~cm}^{-2} \mathrm{sr}^{-1} \mathrm{~cm}$ in channel 1 (Fig. 4a). The continuumlike offset results from upwelling greybody-like emission from the surface and/or tropospheric clouds, which is scattered by the PSC particles. A characteristic "shoulder-like" signature is found in the spectral region around $820 \mathrm{~cm}^{-1}$, which is shaded in light cyan here and in the following. 


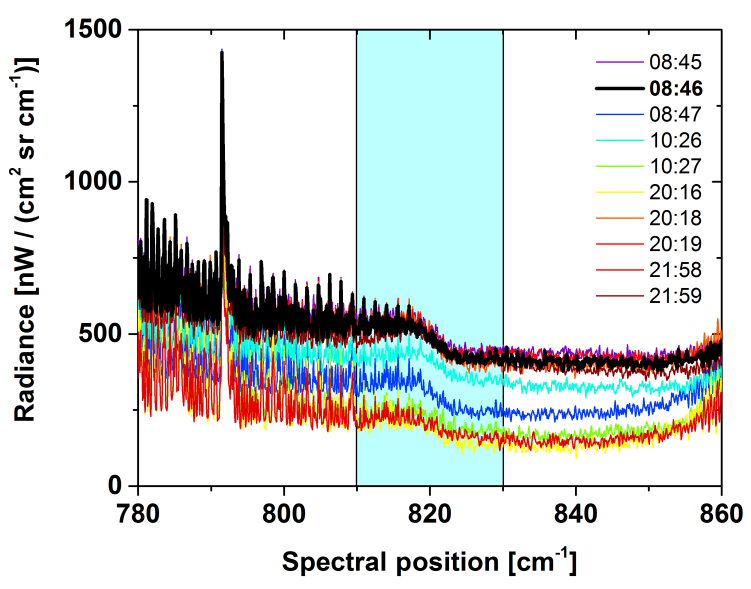

Figure 5. Spectral window of MIPAS-Envisat channel A observations with tangent altitudes between 18.4 and $19.0 \mathrm{~km}$ in the vicinity of the Geophysica flight on 11 December 2011. Geolocations are indicated in Fig. 1. Observation times are given in UTC.

The channel 2 spectra (Fig. 4b) are characterized by lower overall radiances when compared to channel 1 . The spectra show a characteristic continuum-like offset of up to $\sim 150 \times$ $10^{-9} \mathrm{~W} \mathrm{~cm}^{-2} \mathrm{sr}^{-1} \mathrm{~cm}$ at the lower boundary of the channel, which decreases towards higher wavenumbers. Absorption signatures of gaseous $\mathrm{H}_{2} \mathrm{O}$ are found between 1170 and $1250 \mathrm{~cm}^{-1}$ (blue arrows in Fig. $4 \mathrm{~b}$ ) and are the consequence of absorption of upwelling broadband radiation from the surface and/or low clouds by the abundant $\mathrm{H}_{2} \mathrm{O}$ molecules in the lower troposphere (see Höpfner et al., 2002).

Figure 5 shows a spectral window from 780 to $860 \mathrm{~cm}^{-1}$ of MIPAS-Envisat channel A observations in the vicinity of the Geophysica flight exhibiting PSC signatures (from visual inspection) (see Fig. 1). The observations with tangent altitudes close to the Geophysica flight altitude also show a "shoulder-like" signature in the spectral region around $820 \mathrm{~cm}^{-1}$ similar to the MIPAS-STR observations.

\subsection{Ambient conditions and indications of PSC composition}

Figure 6 shows the vertical cross sections of temperature and $\mathrm{HNO}_{3(\mathrm{~g})}$ retrieved from the MIPAS-STR observations. In Appendix A (Fig. A1) we furthermore show the associated vertical cross sections of $\mathrm{H}_{2} \mathrm{O}_{(\mathrm{g})}$ and $\mathrm{O}_{3}$, since these results are used below. Where applicable, the physical state of $\mathrm{HNO}_{3}$ and $\mathrm{H}_{2} \mathrm{O}$ is indicated by "(g)" for "gaseous" and "(s)" for "solid". All other discussed compounds are gaseous under stratospheric conditions, and the physical state is not indicated explicitly.

The vertical cross section of temperature in Fig. 6a typically shows temperatures above $200 \mathrm{~K}$ around flight altitude until $\sim$ 13:00 UTC. From the second half of section $\mathrm{c}$ to section e and in particular during the PSC encounter, lower temperatures between 195 and $200 \mathrm{~K}$ are found above
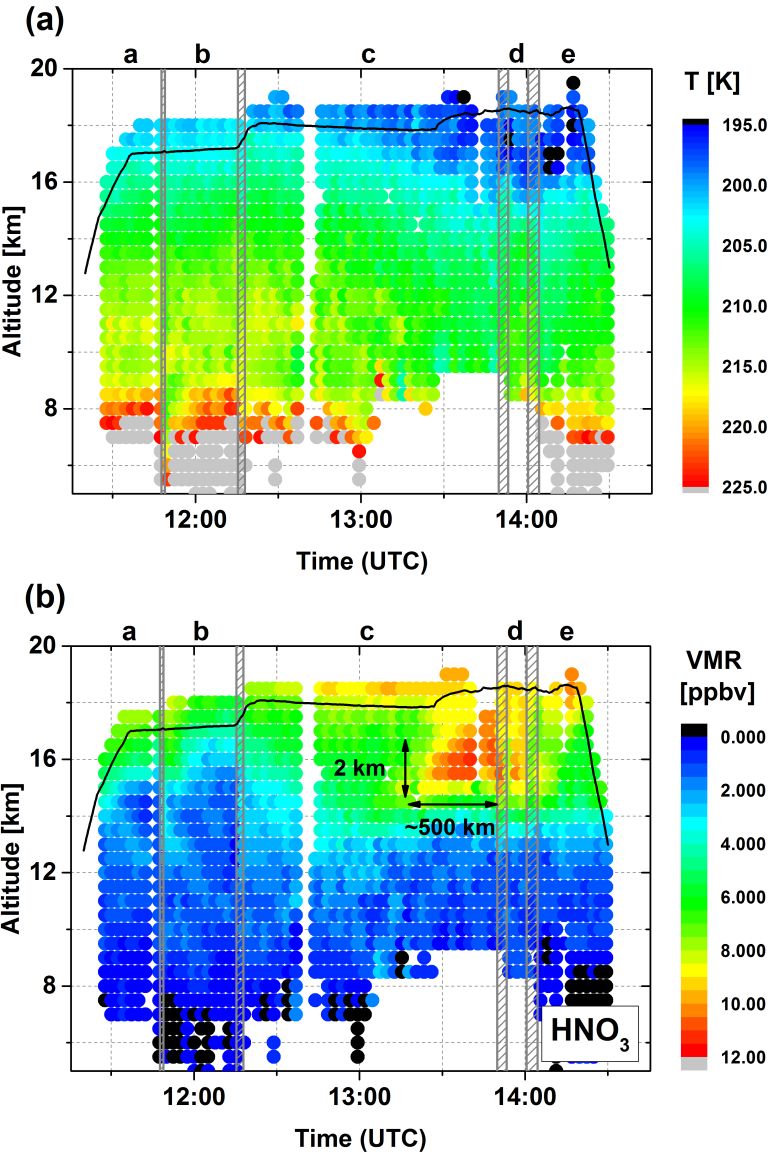

Figure 6. Vertical cross sections of temperature $(T)$ and $\mathrm{HNO}_{3}(\mathrm{~g})$ along the flight track retrieved from the MIPAS-STR observations. Vertical flight profile (black line) and turns between different flight legs (grey hatched areas). Data points are filtered for a vertical resolution better than $5 \mathrm{~km}$.

$16 \mathrm{~km}$. The observed temperatures are close to typical values of $T_{\mathrm{NAT}}$ (Hanson and Mauersberger, 1988) and above the existence temperatures of STS $\left(T_{\mathrm{STS}}, \sim 3.5 \mathrm{~K}\right.$ below $T_{\mathrm{NAT}}$, Drdla et al., 2003) and ice ( $T_{\text {ice }}, \sim 7 \mathrm{~K}$ below $T_{\mathrm{NAT}}$, Murphy and Koop, 2005).

The vertical cross section of $\mathrm{HNO}_{3(\mathrm{~g})}$ shows mixing ratios increasing with altitude in general (Fig. 6b). In the horizontal direction, the mixing ratios around and below flight altitude slightly decrease in section $b$ and increase again at the beginning of section $\mathrm{c}$, indicating the crossing of the vortex edge (compare Fig. 1). Between 13:15 and 14:15 UTC, a local $\mathrm{HNO}_{3(\mathrm{~g})}$ maximum peaking at $\sim 16 \mathrm{~km}$ is observed. The maximum is located below the PSC encounter of the Geophysica (see Sect. 2.1) and extends into the PSC observed at flight altitude. In contrast, the vertical cross section of the stratospheric tracer $\mathrm{O}_{3}$ in Appendix A (Fig. A1) shows no corresponding maximum, suggesting that the $\mathrm{HNO}_{3(\mathrm{~g})}$ maximum originated from nitrification. 


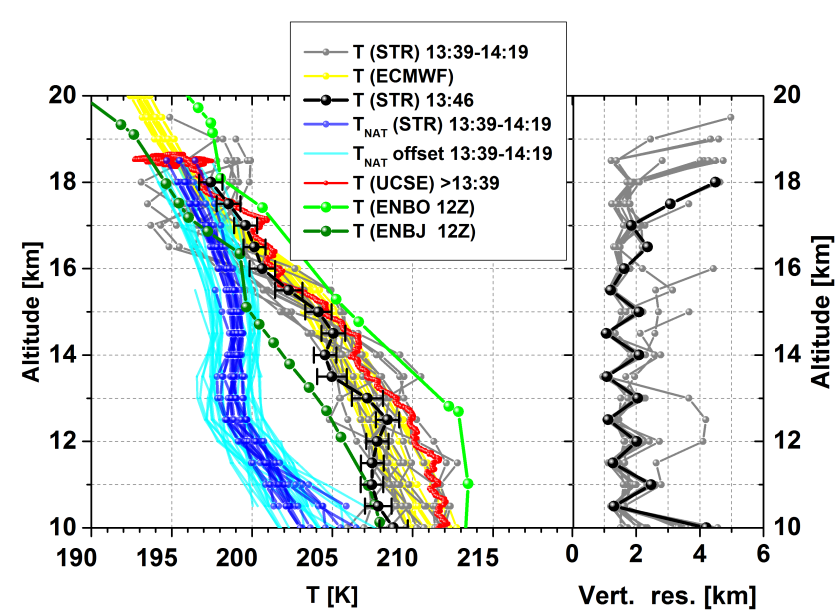

Figure 7. Left panel: MIPAS-STR temperature profiles (grey) associated with the Geophysica PSC encounter. Associated ECMWF initial guess/a priori profiles (yellow). Individual MIPAS-STR temperature profile (black) with combined random and systematic $1 \sigma-$ uncertainties. In situ measurements by the Geophysica UCSE onboard temperature sensor (red) during the PSC encounter and the subsequent descent phase. Radiosonde temperature profiles from Bodø (ENBO, green) and Bjornoya (ENBJ, dark green). $T_{\text {NAT }}$ profiles calculated from the MIPAS-STR $\mathrm{HNO}_{3(\mathrm{~g})}$ and $\mathrm{H}_{2} \mathrm{O}_{(\mathrm{g})}$ profiles during the PSC encounter (blue) together with the same profiles (cyan) considering a simultaneous $+20 \%$ and a simultaneous $-20 \%$ offset for both species, respectively. Right panel: vertical resolutions of MIPAS-STR profiles.

In Fig. 7, we show $T_{\mathrm{NAT}}$ calculated from the MIPAS-STR $\mathrm{HNO}_{3(\mathrm{~g})}$ and $\mathrm{H}_{2} \mathrm{O}_{(\mathrm{g})}$ observations during the PSC encounter. $T_{\mathrm{NAT}}$ is calculated using the thermodynamic relationships of Hanson and Mauersberger (1988). The $T_{\mathrm{NAT}}$ profiles are compared with the corresponding temperature profiles derived from the MIPAS-STR observations, the Geophysica UCSE (Unit for Connection with Scientific Equipment) onboard temperature sensor measurements, radiosonde temperature profiles from Bodø and Bjornoya (12Z launches; see Fig. 1), and ECMWF temperature profiles (here: ECMWF T106 grid-point analysis). The same ECMWF profiles served as the initial guess and a priori profiles for the MIPAS-STR temperature retrievals.

The MIPAS-STR $\mathrm{HNO}_{3(\mathrm{~g})}$ and $\mathrm{H}_{2} \mathrm{O}_{(\mathrm{g})}$ profiles used for the calculation of $T_{\text {NAT }}$ are shown in Appendix A (Fig. A2). The $\mathrm{H}_{2} \mathrm{O}_{(\mathrm{g})}$ profiles are furthermore compared with in situ observations of total $\mathrm{H}_{2} \mathrm{O}$ by FISH (Fast In-situ Stratospheric Hygrometer; Zöger et al., 1999; Meyer et al., 2015) during the same flight for quality assessment.

During the PSC encounter, both MIPAS-STR and the Geophysica UCSE in situ temperature sensor indicate temperatures between 192 and $200 \mathrm{~K}$ around flight altitude. The UCSE temperatures are slightly lower than the temperatures measured by MIPAS-STR. Both observations are consistent with the radiosonde profiles from Bodø (south of the PSC

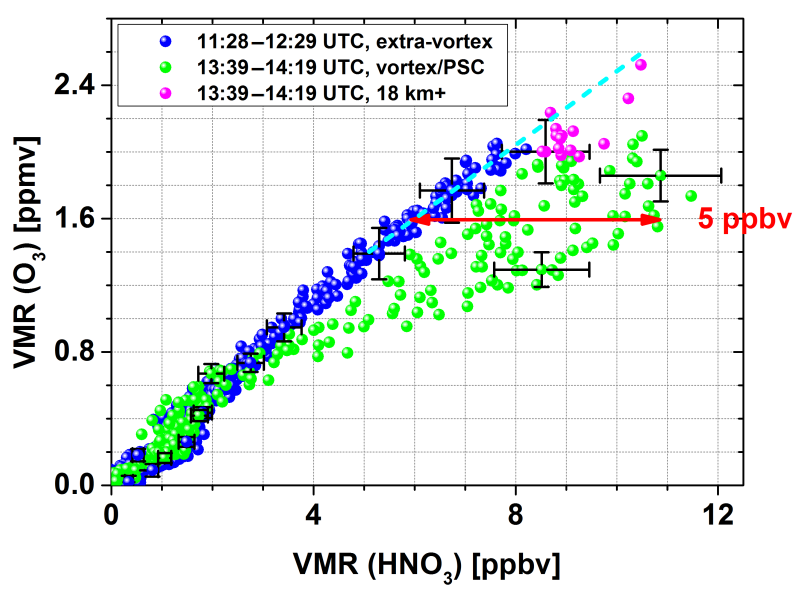

Figure 8. Correlation of $\mathrm{O}_{3}$ with $\mathrm{HNO}_{3}$ (g) retrieved from the MIPAS-STR observations together with combined random and systematic $1 \sigma$-uncertainties of example data points. Blue data points: correlation outside the polar vortex. Green data points: correlation inside the polar vortex and inside/below the observed PSC (data points only where PSC is sufficiently transparent for trace gas retrieval; compare Figs. $6 \mathrm{~b}$ and A1b). Magenta data points are a subset of the vortex data points close to flight altitude $(\geq 18 \mathrm{~km})$ inside the PSC. Cyan dashed line: extrapolation of extra-vortex correlation to higher mixing ratios (i.e., altitudes).

encounter) and Bjornoya (north of the PSC encounter). The ECMWF temperatures are in close agreement with the UCSE data. Slightly higher and more variable temperatures are derived from MIPAS-STR around flight altitude and hint at enhanced uncertainties (e.g., additional errors due to a weak cloud index threshold of 2 applied in the retrievals) and/or horizontal temperature variations around the flight track.

While several MIPAS-STR temperature profiles approach $T_{\mathrm{NAT}}$ values already at altitudes $>16 \mathrm{~km}$, the UCSE in situ and ECMWF temperatures approach $T_{\mathrm{NAT}}$ at $\sim 18 \mathrm{~km}$. The same $T_{\text {NAT }}$ profiles were also calculated considering offsets of (i) $+20 \%$ in both $\mathrm{HNO}_{3(\mathrm{~g})}$ and $\mathrm{H}_{2} \mathrm{O}_{(\mathrm{g})}$ and (ii) $-20 \%$ in both $\mathrm{HNO}_{3(\mathrm{~g})}$ and $\mathrm{H}_{2} \mathrm{O}_{(\mathrm{g})}$ (i.e., offsets for $\mathrm{HNO}_{3(\mathrm{~g})}$ and $\mathrm{H}_{2} \mathrm{O}_{(\mathrm{g})}$ acting in the same direction in terms of $\left.T_{\mathrm{NAT}}\right)$. The offset $T_{\text {NAT }}$ profiles indicate a weak sensitivity of $T_{\text {NAT }}$ to uncertainties in $\mathrm{HNO}_{3(\mathrm{~g})}$ and $\mathrm{H}_{2} \mathrm{O}_{(\mathrm{g})}$. The close agreement of the temperatures measured by MIPAS-STR, the UCSE data, and the ECMWF data with calculated $T_{\mathrm{NAT}}$ around flight altitude suggests that the observed PSC was composed of $\beta$ NAT. NAD is another possible candidate due to similar formation and persistence conditions when compared with $\beta$ NAT (Worsnop et al., 1993; Tizek et al., 2004). $\alpha$-NAT, STS, and ice are unlikely candidates due to too high temperatures.

To investigate the accumulated vertical $\mathrm{HNO}_{3}$ redistribution at the locations inside/below the probed PSC, the correlations of the MIPAS-STR $\mathrm{HNO}_{3(\mathrm{~g})}$ and $\mathrm{O}_{3}$ profiles associated with the PSC encounter (see Fig. A2a and c) and profiles outside the polar vortex are shown in Fig. 8. $\mathrm{O}_{3}$ is well suited as a stratospheric tracer in this case, since the altitude 
range under consideration is hardly affected by Arctic winter $\mathrm{O}_{3}$ depletion at this early stage of the polar winter. Therefore, deviations of the correlation inside the polar vortex and inside/below the PSC (green data points) from the unperturbed correlation outside the polar vortex (blue data points) indicate excess $\mathrm{HNO}_{3(\mathrm{~g})}$ or $\mathrm{HNO}_{3(\mathrm{~g})}$ deficit due to condensation, sedimentation, and evaporation of $\mathrm{HNO}_{3}$-containing particles. The comparison of the correlations shows that inside and below the PSC the retrieved $\mathrm{HNO}_{3(\mathrm{~g})}$ mixing ratios match or exceed the correlation outside the polar vortex. Maximum differences in $\mathrm{HNO}_{3}$ (g) of $\sim 5 \mathrm{ppbv}$ are found for $\mathrm{O}_{3}$ mixing ratios around 1.4 to $1.8 \mathrm{ppmv}$. For the profiles associated with the PSC encounter, this coincides with the altitude range around the nitrification peak at $\sim 16 \mathrm{~km}$ (Fig. 6b). Figure 8 furthermore shows that the vortex correlation around flight altitude and inside the PSC (magenta data points) matches or slightly exceeds the extra-vortex correlation extrapolated to higher altitudes (cyan dashed line). This suggests that any $\mathrm{HNO}_{3}$ (s) condensed in the PSC particles represents excess $\mathrm{HNO}_{3}$ originating from particle sedimentation from higher altitudes. Denitrified conditions (i.e., data points on the left side of the extra-vortex correlation) are not found in the altitude range covered by the MIPAS-STR observations.

\section{Analysis of PSC signatures in the MIPAS-STR spectra}

\subsection{Radiative transfer model and simulation scenario}

To simulate the MIPAS-STR spectra, we use the Karlsruhe Optimized and Precise Radiative Transfer Algorithm (KOPRA, Stiller et al., 2002). KOPRA is a fast line-by-line code and allows the modeling of infrared limb observations, including trace gas signatures, cloud parameters, and instrumental characteristics. Modeling of MIPAS PSC observations using a Mie model and based on single scattering is discussed and validated by Höpfner et al. (2002, 2006a), Höpfner (2004), and Höpfner and Emde (2004), respectively. In the following, we consider the MIPAS-STR limb view at 13:47 UTC in approximately horizontal orientation (black spectra in Fig. 4a and b), which was performed well within the PSC and shows developed PSC signatures. Table 1 provides an overview of the radiative transfer scenarios discussed in the following. To simulate the signatures of $\beta$-NAT, we use the "NATcoa" refractive indices by Biermann (1998), which have been applied successfully for the identification for small $\beta$-NAT particles by Höpfner et al. (2006a, b). Hereafter, we refer with $\beta$-NAT to the "NATcoa" refractive indices by Biermann et al. (1998). To simulate the signatures of $\alpha$-NAT, we use the refractive indices for $\alpha$-NAT aerosols by Richwine et al. (1995), which show a more developed signature around $820 \mathrm{~cm}^{-1}$ than the corresponding faint signature in the refractive indices for $\alpha$-NAT by Toon et al. (1994). (a)


Figure 9. (a) Particle size distributions derived from the FSSP100 (black) and CDP (magenta) measurements during the PSC encounter (from Molleker et al., 2014, Fig. 12). (b) Particle size distributions used in radiative transfer simulations of MIPAS-STR PSC spectra (red and blue) and simulated by the CLaMS (grey). Note that particle sizes are indicated in diameter $(D)$.

The signatures of NAD are simulated using the refractive indices by Niedzela et al. (1998), which closely correspond to spectroscopic data of $\alpha$-NAD (Grothe et al., 2004). To simulate STS, we use the refractive indices by Biermann et al. (2000) for $45 \mathrm{wt} \%$ solutions of $\mathrm{HNO}_{3} / \mathrm{H}_{2} \mathrm{O}$, since these compounds are expected to dominate the optical properties of STS (Höpfner et al., 2006a). For ice, we use the refractive indices by Zasetsky et al. (2005) for $200 \mathrm{~K}$.

Particle size distributions are assumed to be lognormal in shape and characterized by the mode radii $(r)$, geometric standard deviations (i.e., mode width, $\sigma$ ), and mode number densities $(N)$. For simplification, we assume that these quantities are constant over the entire vertical range covered by the PSC. We constrain the used size distributions with the FSSP-100 and CDP in situ observations during the same 
Table 1. Overview of radiative transfer scenarios.

\begin{tabular}{|c|c|c|c|c|c|c|}
\hline ID & Species & $\begin{array}{l}\text { Size } \\
\text { distribution }\end{array}$ & $\begin{array}{l}\text { Code / aspect } \\
\text { ratio (AR) }\end{array}$ & $\begin{array}{r}\text { Vertical PSC } \\
\text { extent }(\mathrm{km})\end{array}$ & $\begin{array}{l}\text { Vertical range of trop. } \\
\text { cloud }(\mathrm{km}) / \alpha\left(\mathrm{km}^{-1}\right)\end{array}$ & Comment \\
\hline 43 & $\beta$-NAT & $\mathrm{B}$ & $\mathrm{Mie}^{\mathrm{a}} / 1.0$ & $17-23$ & $0-10 / 0.0223$ & \\
\hline $43 a$ & $\beta$-NAT & $\mathrm{B}$ & $\mathrm{Mie}^{\mathrm{a}} / 1.0$ & $17-23$ & $0-10 / 0.0223$ & No scattering \\
\hline 44 & $\beta$-NAT & A & $\mathrm{Mie}^{\mathrm{a}} / 1.0$ & $17-23$ & $0-10 / 0.0223$ & \\
\hline 45 & STS & $\mathrm{B}$ & $\mathrm{Mie}^{\mathrm{a}} / 1.0$ & $17-23$ & $0-10 / 0.0223$ & \\
\hline 46 & STS & $\mathrm{A}$ & $\mathrm{Mie}^{\mathrm{a}} / 1.0$ & $17-23$ & $0-10 / 0.0223$ & \\
\hline 47 & NAD & $\mathrm{B}$ & $\mathrm{Mie}^{\mathrm{a}} / 1.0$ & $17-23$ & $0-10 / 0.0223$ & \\
\hline 48 & NAD & A & $\mathrm{Mie}^{\mathrm{a}} / 1.0$ & $17-23$ & $0-10 / 0.0223$ & \\
\hline 49 & $\alpha$-NAT & $\mathrm{B}$ & $\mathrm{Mie}^{\mathrm{a}} / 1.0$ & $17-23$ & $0-10 / 0.0223$ & \\
\hline 50 & $\alpha$-NAT & A & $\mathrm{Mie}^{\mathrm{a}} / 1.0$ & $17-23$ & $0-10 / 0.0223$ & \\
\hline 51 & Ice & $\mathrm{B}$ & $\mathrm{Mie}^{\mathrm{a}} / 1.0$ & $17-23$ & $0-10 / 0.0223$ & \\
\hline 52 & Ice & A & $\mathrm{Mie}^{\mathrm{a}} / 1.0$ & $17-23$ & $0-10 / 0.0223$ & \\
\hline 53 & $\beta$-NAT & B & $\mathrm{TM}^{\mathrm{b}} / 0.5$ & $17-23$ & $0-10 / 0.0223$ & \\
\hline 54 & $\beta$-NAT & A & $\mathrm{TM}^{\mathrm{b}} / 0.5$ & $17-23$ & $0-10 / 0.0223$ & \\
\hline 55 & $\beta$-NAT & $\mathrm{B}$ & $\mathrm{TM}^{\mathrm{b}} / 2.0$ & $17-23$ & $0-10 / 0.0223$ & \\
\hline 56 & $\beta$-NAT & A & $\mathrm{TM}^{\mathrm{b}} / 2.0$ & $17-23$ & $0-10 / 0.0223$ & \\
\hline 57 & $\beta$-NAT & B & $\mathrm{TM}^{\mathrm{c}} / 0.1$ & $17-23$ & $0-10 / 0.0223$ & \\
\hline $57 \mathrm{a}$ & $\beta$-NAT & $\mathrm{B}$ & $\mathrm{TM}^{\mathrm{c}} / 0.1$ & $17-23$ & $0-10 / 0.0223$ & No scattering \\
\hline $57 \mathrm{~b}$ & $\beta$-NAT & $\mathrm{B}$ & $\mathrm{TM}^{\mathrm{c}} / 0.1$ & $17-23$ & $0-2 / 5.000$ & \\
\hline $57 \mathrm{c}$ & $\beta$-NAT & $\mathrm{B}$ & $\mathrm{TM}^{\mathrm{c}} / 0.1$ & $17-23$ &.$/$ & \\
\hline $57 d$ & $\beta$-NAT & $\mathrm{B}$ & $\mathrm{TM}^{\mathrm{c}} / 0.1$ & $17-23$ & $0-10 / 5.000$ & \\
\hline $57 \mathrm{e}$ & $\beta$-NAT & $\mathrm{B}$ & $\mathrm{TM}^{\mathrm{c}} / 0.1$ & $17-23$ & $0-10 / 0.0223$ & $T_{\text {Surface }}=273.2 \mathrm{~K}$ \\
\hline $57 \mathrm{f}$ & $\beta$-NAT & $\mathrm{B}$ & $\mathrm{TM}^{\mathrm{c}} / 0.1$ & $17-20$ & $0-10 / 0.0223$ & \\
\hline $57 \mathrm{~g}$ & $\beta$-NAT & B1 & $\mathrm{TM}^{\mathrm{c}} / 0.1$ & $17-23$ & $0-10 / 0.0223$ & \\
\hline $57 \mathrm{i}$ & $\beta$-NAT & B2 & $\mathrm{TM}^{\mathrm{c}} / 0.1$ & $17-23$ & $0-10 / 0.0223$ & \\
\hline 58 & $\beta$-NAT & A & $\mathrm{TM}^{\mathrm{c}} / 0.1$ & $17-23$ & $0-10 / 0.0223$ & \\
\hline 59 & $\beta$-NAT & $\mathrm{B}$ & $\mathrm{TM}^{\mathrm{c}} / 10.0$ & $17-23$ & $0-10 / 0.0223$ & \\
\hline 60 & $\beta$-NAT & A & $\mathrm{TM}^{\mathrm{c}} / 10.0$ & $17-23$ & $0-10 / 0.0223$ & \\
\hline
\end{tabular}

${ }^{a}$ Mie code, Höpfner et al. (2006a). ${ }^{b}$ EBCM T-Matrix code, Mishchenko and Travis (1998). ${ }^{c}$ IIM + SOV T-Matrix code, Bi et al. (2013).

Table 2. Particle size distributions used in radiative transfer scenarios.

\begin{tabular}{lrrrrrrr}
\hline Size & \multicolumn{3}{c}{ Mode 1} & & \multicolumn{3}{c}{ Mode 2 } \\
\cline { 2 - 4 } \cline { 6 - 8 } distribution & $r_{1}(\mu \mathrm{m})$ & $\sigma_{1}$ & $N_{1}\left(\mathrm{~cm}^{-3}\right)$ & & $r_{2}(\mu \mathrm{m})$ & $\sigma_{2}$ & $N_{2}\left(\mathrm{~cm}^{-3}\right)$ \\
\hline A & 2.5 & 1.5 & $1.6 \times 10^{-3}$ & & 6.0 & 1.35 & $1.5 \times 10^{-3}$ \\
$\mathrm{~B}$ & 2.0 & 1.5 & $1.6 \times 10^{-3}$ & & 4.8 & 1.35 & $1.5 \times 10^{-3}$ \\
$\mathrm{~B} 1$ &.$/$ &.$/$ &. & & 4.8 & 1.35 & $1.5 \times 10^{-3}$ \\
$\mathrm{~B} 2$ & 0.5 & 1.5 & $1.6 \times 10^{-1}$ & & 4.8 & 1.35 & $1.5 \times 10^{-3}$ \\
\hline
\end{tabular}

flight. In Fig. 9a, the particle size distributions derived by Molleker et al. (2014) from the FSSP-100 and CDP observations (black and magenta, respectively) during the PSC encounter are shown. Figure 9b shows the lognormal size distributions used in the radiative transfer simulations of the MIPAS-STR observations (for parameters, see Table 2). The bimodal size distribution A (Fig. 9b, red) is adjusted manually to match the size distribution derived from the FSSP-100 observations in terms of shape and condensed $\mathrm{HNO}_{3}$.
To improve the agreement of the simulated spectra with the measurements, only the mode radii of size distribution A are scaled. Accordingly, the particle number densities and mode widths are maintained, while the particle sizes and thereby the volume of $\mathrm{HNO}_{3(\mathrm{~s})}$ are scaled. In Fig. 9b, size distribution $\mathrm{B}$ corresponds to the size distribution, resulting in the best agreement of the radiative transfer simulations with the MIPAS-STR observations (see below). Size distribution B is obtained from size distribution A by scaling the 
radii of both modes by a factor of 0.8 . Scenarios B1 and B2 are sensitivity calculations with respect to the importance of the larger mode and a hypothetic small particle mode with enhanced number density.

For modeling of aspherical particles, spheroids are assumed. The aspect ratio (AR) is the ratio of the horizontal and rotational semi-axis. Elongated spheroids are characterized by $\mathrm{AR}<1$, and oblate spheroids by $\mathrm{AR}>1$. For easy comparisons, the sizes of aspherical particles are given in $r$ or $D$ of volume-equivalent spherical particles.

In the simulations, we adopt a vertical PSC extent from 17 to $23 \mathrm{~km}$ altitude, which is supported by the MIPAS-STR and CALIPSO observations (see Sect. 2.2 and 2.3). The tropospheric cloud scenario is defined considering the MIPASSTR cloud index values of the associated limb scan. Further information on the tropospheric cloud scenario is inferred from the fact that tropospheric $\mathrm{H}_{2} \mathrm{O}_{(\mathrm{g})}$ absorption signatures are identified in the MIPAS-STR spectra (see Fig. 4b). While the MIPAS-STR cloud index values suggest a tropospheric cloud top slightly below $10 \mathrm{~km}$ (see Fig. 2), the presence of the $\mathrm{H}_{2} \mathrm{O}_{(\mathrm{g})}$ absorption lines requires partially transparent conditions down to low tropospheric altitudes (Höpfner et al., 2002). Both criteria are fulfilled by assuming a partially transparent tropospheric cloud from 0 to $10 \mathrm{~km}$ characterized by a low continuum extinction coefficient $\alpha$ of $0.0223 \mathrm{~km}^{-1}$ (i.e., $\sim 98 \%$ transmission per $1 \mathrm{~km}$ layer), providing efficient transmission of radiation from nadir directions and increasing opaqueness towards limb geometry at altitudes covered by the tropospheric clouds. Note however that this is a simplified assumption for a probably much more complex and horizontally variable cloud scenario in reality.

A sea surface temperature of $\sim 280.2 \mathrm{~K}$ is adopted as surface temperature from the NOAA Optimum Interpolation (OI) SST V2 GraDS image of the flight day (see http: //www.esrl.noaa.gov/psd/) in combination with a sea surface emissivity of 0.99 (see Newman et al., 2005).

Vertical profiles of trace gases required for the simulations of the spectra are retrieved previously from the full cloud-filtered MIPAS-STR limb scans. The retrievals of temperature (utilizing $\mathrm{CO}_{2}$ signatures), $\mathrm{O}_{3}, \mathrm{HNO}_{3(\mathrm{~g})}, \mathrm{ClONO}_{2}$, CFC-11, and CFC-12 are performed according to Woiwode et al. (2012, 2015). $\mathrm{H}_{2} \mathrm{O}_{(\mathrm{g})}, \mathrm{N}_{2} \mathrm{O}, \mathrm{CH}_{4}, \mathrm{COF}_{2}$, CFC-22, and $\mathrm{CCl}_{4}$ are retrieved from the MIPAS-STR channel 1 and 2 spectra in specific microwindows using the same strategy. As the MIPAS-STR limb observations provide only information above the tropospheric cloud top, the profiles are extrapolated to lower altitudes based on the slopes of the a priori profiles. The profiles of temperature and the strong tropospheric absorber $\mathrm{H}_{2} \mathrm{O}_{(\mathrm{g})}$ below the tropospheric cloud top are constructed from the radiosonde observations from Bod $\varnothing$ and Bjornoya (see Fig. 1). For $\mathrm{CO}_{2}$, the corresponding polar winter profile for MIPAS of Remedios et al. (2007) is updated for the polar winter 2011/12. For the minor constituents $\mathrm{SF}_{6}$, CFC-14, and $\mathrm{N}_{2} \mathrm{O}_{5}$, the polar winter profiles from Remedios et al. (2007) are adopted.

\subsection{Mie calculations of spherical $\beta$-NAT, $\alpha$-NAT, NAD, STS, and ice particles}

For simulations of the ensemble-averaged single scattering parameters of spherical $\beta$-NAT, $\alpha$-NAT, NAD, STS, and ice particles, we use the Mie model coupled to KOPRA (Höpfner et al., 2002, 2006a; Höpfner, 2004). The Mie model provides the wavenumber-dependent ensemble-averaged phase functions, extinction cross sections and single scattering albedos required for the simulation of the scattering and absorption/emission of radiation by the particles.

Figure 10 shows the results of the Mie calculations based on size distributions A and B. The upper panels show the simulated spectra together with the observed MIPAS-STR channel 1 and 2 spectra. The residuals between simulation and observation are shown in the lower panels. For size distribution A, the Mie calculations of $\beta$-NAT show systematically higher radiances in channel 1 by typically about 50 to $100 \times 10^{-9} \mathrm{~W} \mathrm{~cm}^{-2} \mathrm{sr}^{-1} \mathrm{~cm}$ when compared to the measurement (Fig. 10a and b, red). The residual values decrease towards the lower and upper boundaries of the channel. A characteristic dip is found in the spectral region around $820 \mathrm{~cm}^{-1}$, where the "shoulder-like" signature is located in the observations.

Enhanced "line-like" residuals in the densely populated spectral regions below $810 \mathrm{~cm}^{-1}$ (mostly $\mathrm{CO}_{2}$ and $\mathrm{O}_{3}$ ), between 850 and $920 \mathrm{~cm}^{-1}\left(\mathrm{HNO}_{3(\mathrm{~g})}\right)$, as well as above $970 \mathrm{~cm}^{-1}\left(\mathrm{O}_{3}\right)$, are explained by uncertainties in the knowledge of the profiles of the corresponding trace gases at tropospheric altitudes and above the flight path. The uncertainties of these parts of the profiles are translated into the simulated spectra by the simulated scattering of radiation by the particles from outside into the field-of-view. Further sources of uncertainties are limitations in the simulated scattering scenario (e.g., a limited number of scattering angles). Furthermore, the noise equivalent spectral radiance of the MIPASSTR spectra steeply increases towards the channel boundaries and enhances the variability in the residuals in these regions.

In channel 2, the simulated radiances are systematically lower by $\sim 25 \times 10^{-9} \mathrm{~W} \mathrm{~cm}^{-2} \mathrm{sr}^{-1} \mathrm{~cm}$ below $1300 \mathrm{~cm}^{-1}$ and show better agreement with the measurements at higher wavenumbers for size distribution A. Similar to the observation, prominent $\mathrm{H}_{2} \mathrm{O}_{(\mathrm{g})}$ absorption signatures are found between 1170 and $1250 \mathrm{~cm}^{-1}$ in the simulation (indicated as black arrows in Figs. 10 to 12). Further "line-like" residuals exceeding the noise level are again explained by uncertainties in the knowledge of the profiles of the corresponding trace gases at tropospheric altitudes and above the flight path (here mostly $\mathrm{O}_{3}, \mathrm{~N}_{2} \mathrm{O}, \mathrm{CH}_{4}$, and $\mathrm{HNO}_{3}$ ), limitations in the simulated scattering scenario, and increasing noise towards the channel boundaries.

The results for size distribution B (Fig. 10a and b, blue) show improved agreement in channel 1 . However, a dip is observed again in the spectral region around $820 \mathrm{~cm}^{-1}$, and the 
(a)

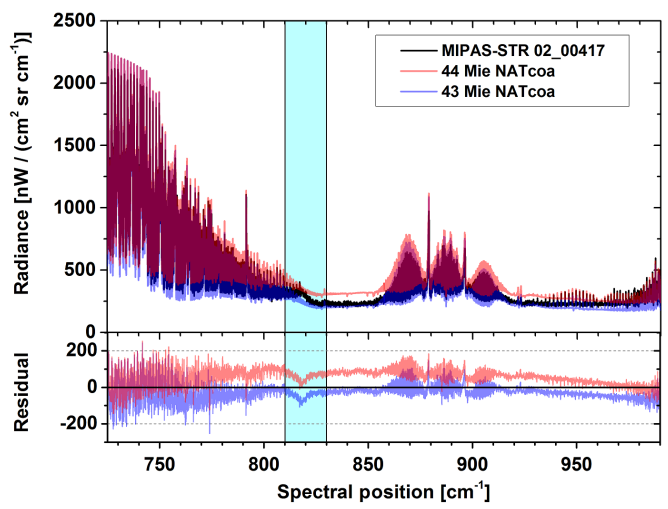

(c)

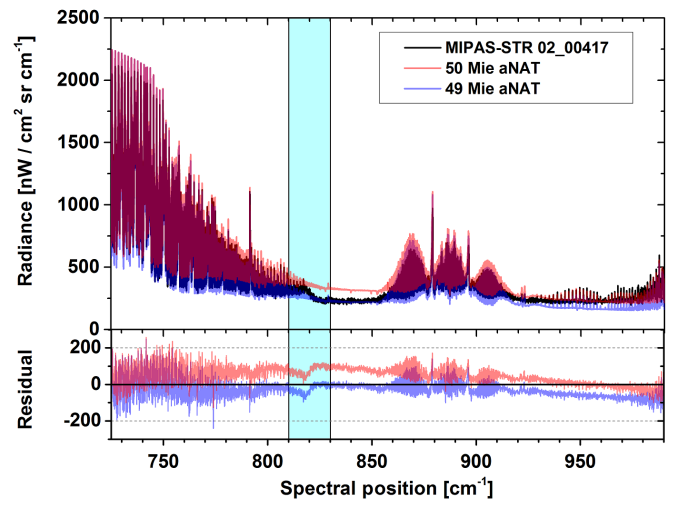

(e)

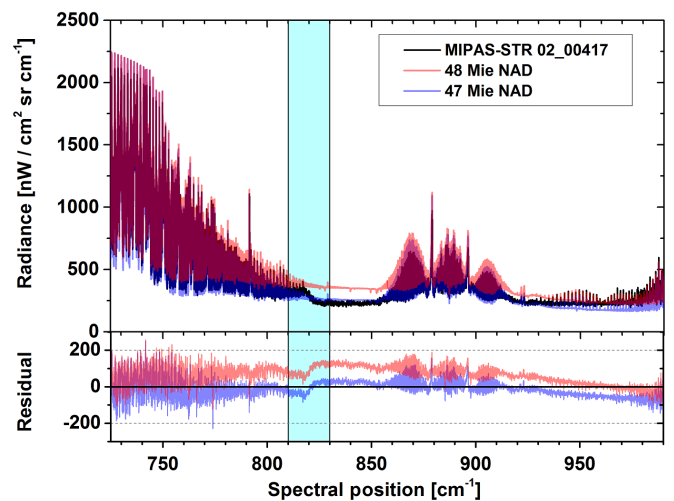

(g)

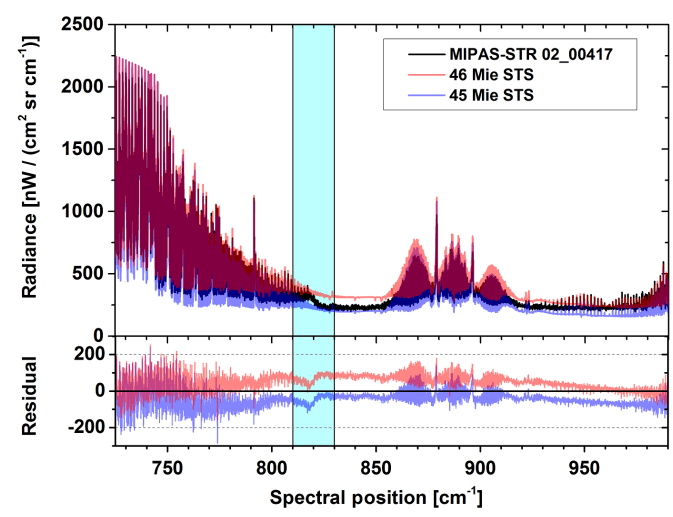

(b)

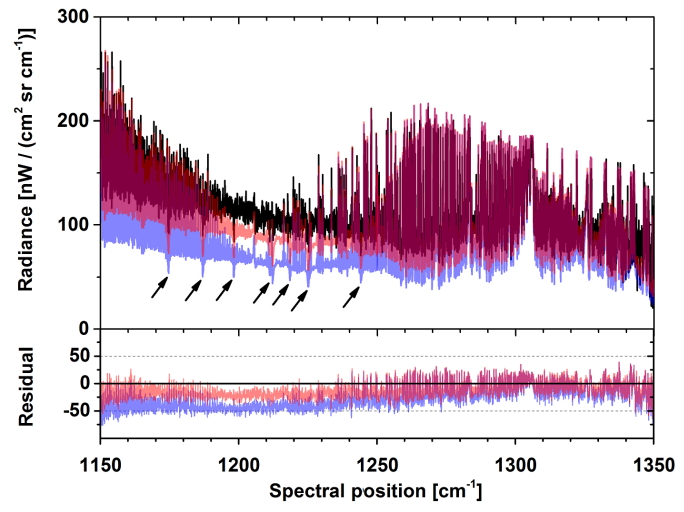

(d)

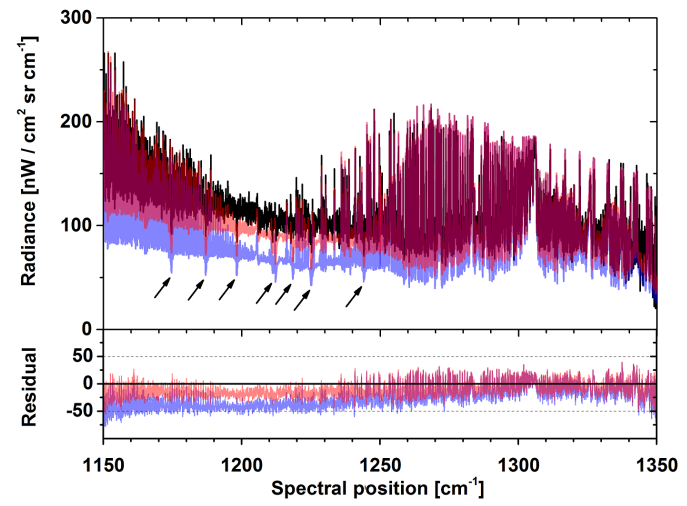

(f)

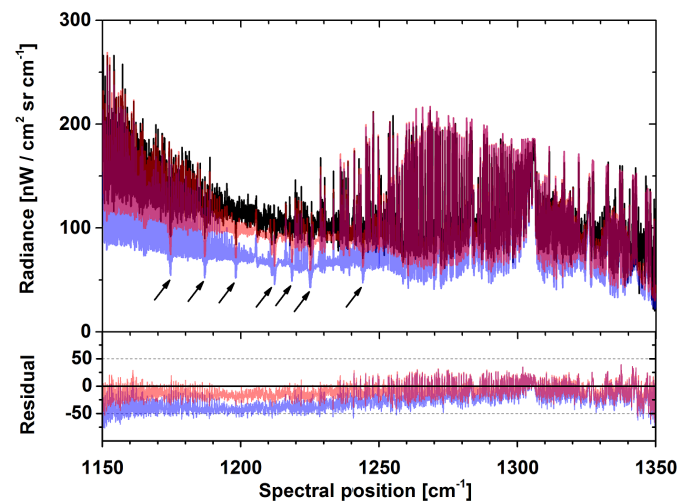

(h)

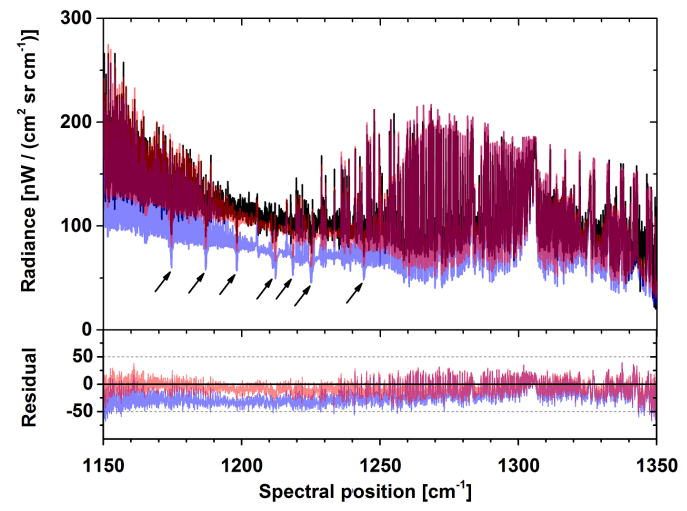

Figure 10. 
(i)

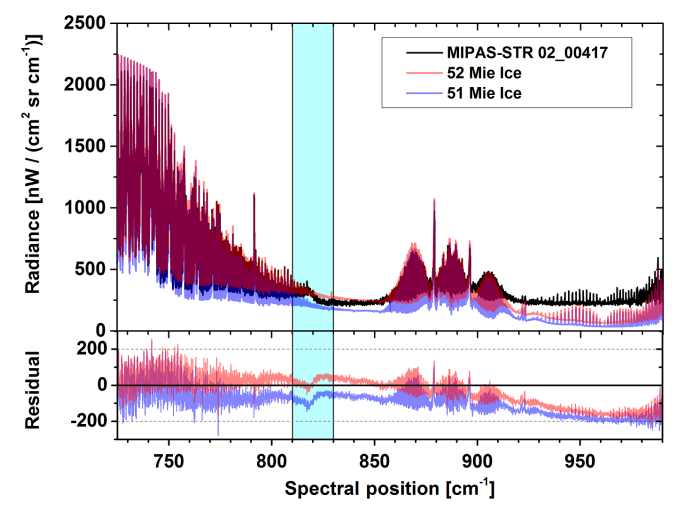

(j)

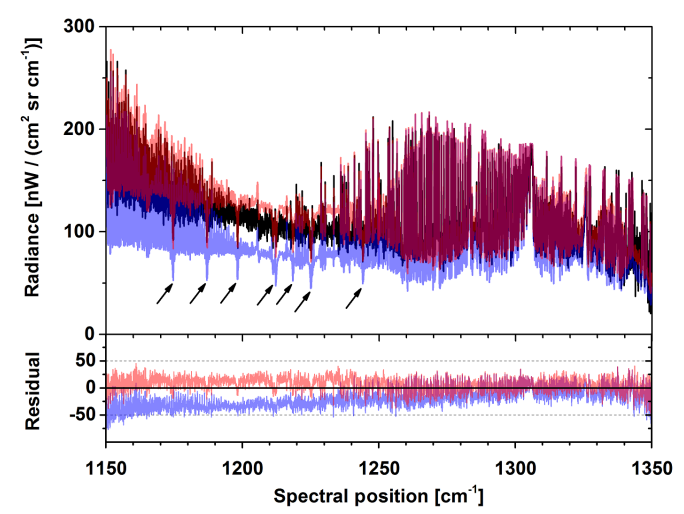

Figure 10. Mie calculations for spherical particles. Black spectra in all panels: MIPAS-STR limb observation (13:47 UTC, tangent altitude $18.4 \mathrm{~km})$. Simulations of (a, b) $\beta$-NAT, $(\mathbf{c}, \mathbf{d}) \alpha$-NAT, $(\mathbf{e}, \mathbf{f})$ NAD, $(\mathbf{g}, \mathbf{h})$ STS, and (i, j) ice with size distributions A (red) and B (blue). Lower panels: residuals between the simulations and the observation. Black arrows denote prominent $\mathrm{H}_{2} \mathrm{O}$ absorption signatures from scattering of tropospheric radiation by PSC particles. Numbers in the legend refer to scenarios in Table 1. "NATcoa" corresponds to $\beta$-NAT and "Mie" to Mie simulation $(\mathrm{AR}=1.0)$.

residual values are increasingly negative towards the lower and upper boundaries of channel 1 . In channel 2 , the residual values below $1300 \mathrm{~cm}^{-1}$ are more negative when compared to size distribution A.

For $\alpha$-NAT (Fig. 10c and d), NAD (Fig. 10e and f), and STS (Fig. 10g and h), similar residuals are found for both size distributions when compared to $\beta$-NAT. For these species, more "step-like" residual signatures are found in the spectral region around $820 \mathrm{~cm}^{-1}$ for both size distributions when compared to $\beta$-NAT. When compared with the previous cases, the simulation of STS using size distribution A results in the best agreement with the observations in channel 2 (Fig. 10h, red).

For ice (Fig. 10i and j), the worst overall agreement is found in channel 1. For both size distributions, the simulated spectrum shows a negative overall gradient from the lower to the upper boundary of the channel. Negative residual values as low as $-200 \times 10^{-9} \mathrm{~W} \mathrm{~cm}^{-2} \mathrm{sr}^{-1} \mathrm{~cm}$ are found at the upper end of channel 1 for both size distributions, and again a "step-like" dip is found in the spectral region around $820 \mathrm{~cm}^{-1}$. In channel 2 , the observed radiances are slightly overestimated below $\sim 1250 \mathrm{~cm}^{-1}$ for size distribution $A$ and significantly underestimated below $1300 \mathrm{~cm}^{-1}$ for size distribution B.

Overall, the results of the Mie simulations show worse agreement with the observations and prominent residual signatures in the spectral region around $820 \mathrm{~cm}^{-1}$. In the following simulations of aspherical particles, we exclude $\alpha$-NAT, NAD, STS, and ice as dominating constituents of the observed PSC for the following reasons.

- $\beta$-NAT exhibits a characteristic spectral signature around $820 \mathrm{~cm}^{-1}$ and is the only PSC constituent known to be thermodynamically stable under the conditions of the flight.

- The metastable $\alpha$-NAT modification also exhibits a characteristic spectral signature around $820 \mathrm{~cm}^{-1}$ with a weaker amplitude (Höpfner et al., 2006a). However, $\alpha$ NAT is expected to irreversibly transform into $\beta$-NAT at the temperatures around flight altitude (Tizek et al., 2004; compare Fig. 7). Furthermore, laboratory experiments by Grothe et al. (2006) support approximately spherical geometries of $\alpha$-NAT particles, while the presented Mie calculations for spherical $\alpha$-NAT particles result only in coarse agreement with the observations.

- While $\alpha$-NAD shows a similar spectral signature with weaker amplitude centered at $808 \mathrm{~cm}^{-1}$ (Niedziela et al., 1998, Grothe et al., 2004), the signature is not capable of reproducing the residual dip slightly below $820 \mathrm{~cm}^{-1}$ (see Sect. 3.5). The same is expected for the high-temperature modification $\beta$-NAD, which was characterized by Grothe et al. (2004) in laboratory experiments and shows a similar spectral signature centered at $811 \mathrm{~cm}^{-1}$. Furthermore, the observations indicate temperatures close to the threshold temperature of $\beta$-NAT and slightly too warm for NAD under stratospheric conditions.

- STS droplets are not expected to grow to the large sizes observed in situ and are not supported by the observed temperatures (e.g., Peter, 1997). Furthermore, STS droplets are expected to be approximately spherical. However, the presented Mie calculations for spherical STS droplets result only in coarse agreement with the observations. 
(a)

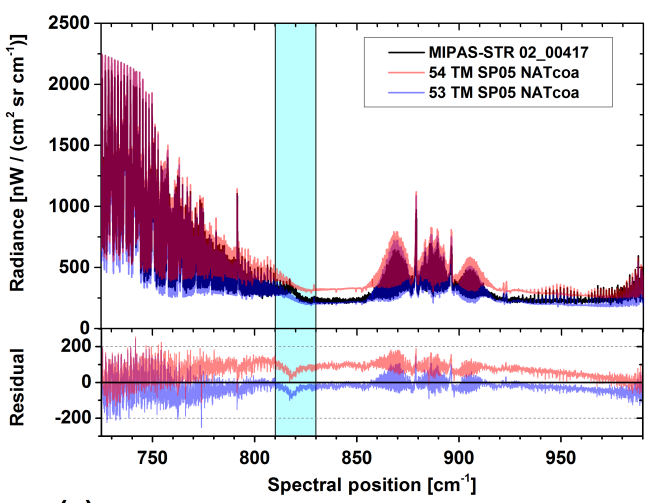

(c)

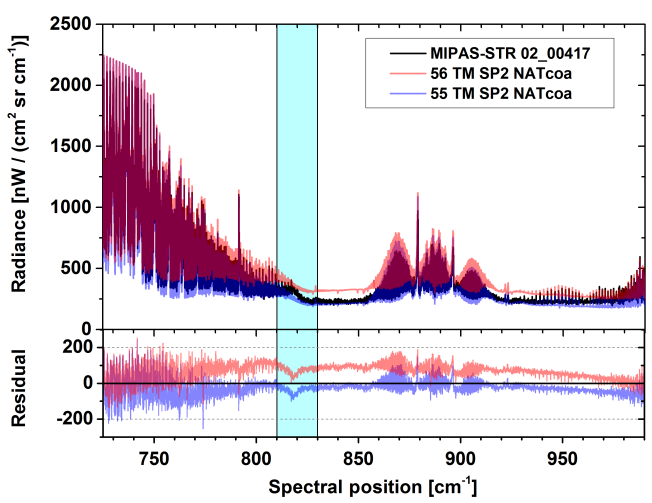

(b)

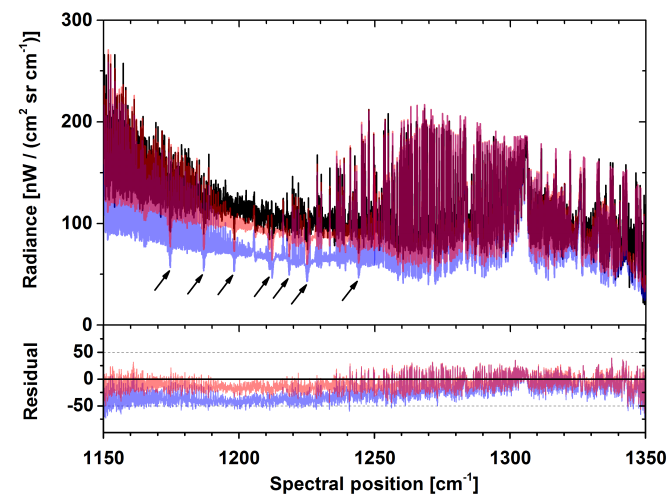

(d)

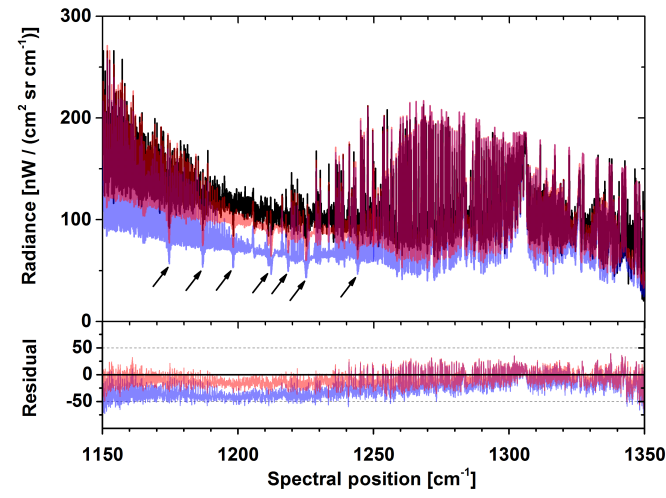

Figure 11. T-Matrix calculations for moderately aspherical $\beta$-NAT particles. Black spectra in all panels: MIPAS-STR limb observation (13:47 UTC, tangent altitude $18.4 \mathrm{~km})$. Simulations of $\beta$-NAT with $(\mathbf{a}, \mathbf{b}) \mathrm{AR}=0.5$ and $(\mathbf{c}, \mathbf{d}) \mathrm{AR}=2.0$ with size distributions $\mathrm{A}$ (red) and $\mathrm{B}$ (blue). Lower panels: residuals between the simulations and the observation. Black arrows denote prominent $\mathrm{H}_{2} \mathrm{O}$ absorption signatures from scattering of tropospheric radiation by PSC particles. Numbers in the legend refer to scenarios in Table 1. "NATcoa" corresponds to $\beta$-NAT, "TM" to T-matrix simulation, and "SP" to spheroid (numbers indicate AR).

- For ice, the observed temperatures are considerably too warm, and the Mie calculations result in the worst overall agreement.

\subsection{T-Matrix calculations of $\beta$-NAT particles with moderate aspect ratios}

For simulations of the ensemble-averaged single scattering parameters of moderately aspherical $\beta$-NAT particles, we use the double-precision T-Matrix code for polydisperse, randomly oriented, rotationally symmetric particles by Mishchenko and Travis (1998). The T-Matrix is calculated according to the extended boundary condition method (EBCM) (Waterman, 1971). Advantages of this code are fast computations for moderately aspherical particles and the capability of direct coupling to KOPRA. The same setup as in the Mie calculations is used.

The results for elongated spheroids with $\mathrm{AR}=0.5$ and oblate spheroids with $\mathrm{AR}=2.0$ are shown in Fig. 11. Briefly, the results for both AR values are very similar to each other and to the Mie scenario (compare Fig. 10a and b) for both size distributions. Again, a characteristic dip is found in the spectral region around $820 \mathrm{~cm}^{-1}$ for both AR values and size distributions.

\subsection{T-Matrix calculations of $\beta$-NAT particles with extreme aspect ratios}

For simulations of the ensemble-averaged single scattering parameters of highly aspherical $\beta$-NAT particles, we use invariant imbedding and separation of variables (IIM + SOV) T-Matrix calculations (Bi et al., 2013). Again, polydisperse, randomly oriented spheroids and the same setup as in the previous scenarios are considered. To allow flexible variation of size distributions and save computing time, the optical parameters of $\beta$-NAT particles are calculated for discrete wavenumbers $v\left(\Delta v=1 \mathrm{~cm}^{-1}\right)$ and sizes $(r=0.5$ to $20 \mu \mathrm{m}$ with $\Delta r=0.5 \mu \mathrm{m})$ and are tabulated. Particle size distributions are implemented by means of binned lognormal size distributions referring to the tabulated optical parameters. The choice of the AR values is motivated by the laboratory experiments by Grothe et al. (2006), resulting in needles 
(a)

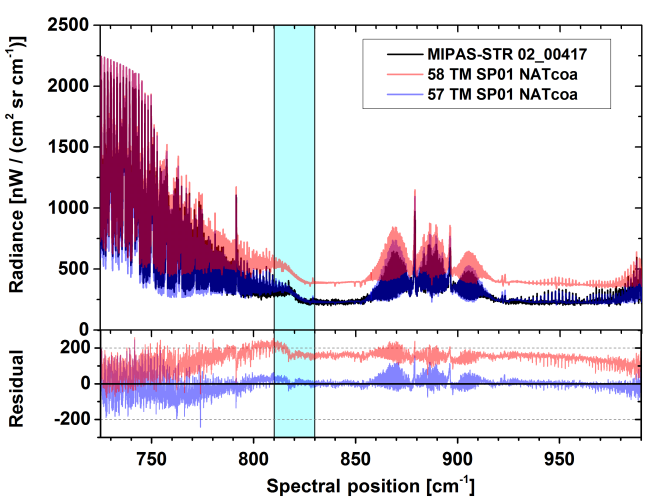

(c)

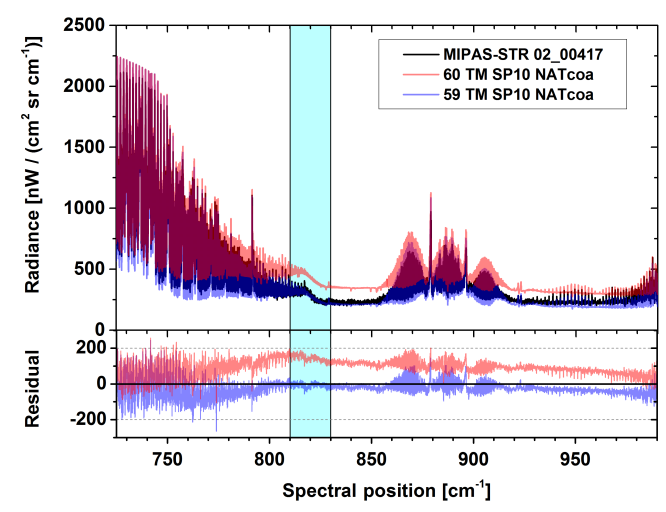

(b)

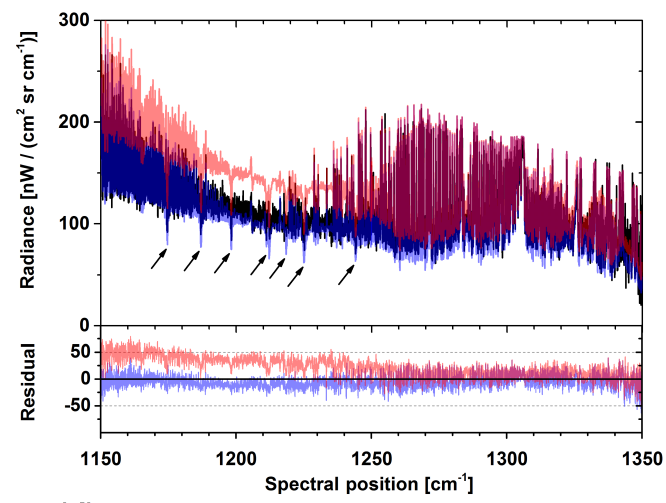

(d)

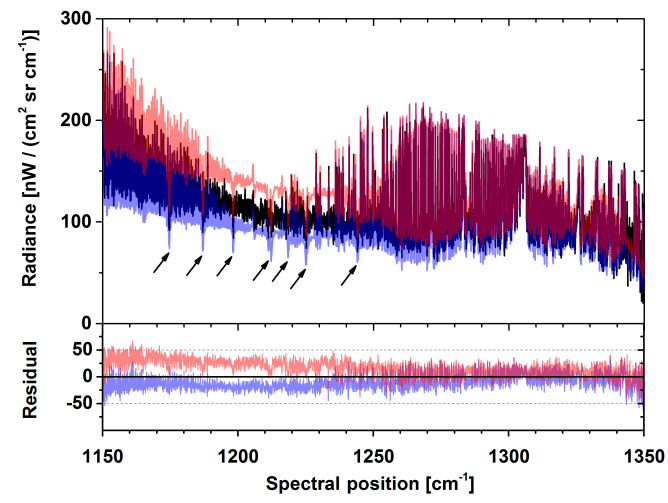

Figure 12. T-Matrix calculations for highly aspherical $\beta$-NAT particles. Black spectra in all panels: MIPAS-STR limb observation (13:47 UTC, tangent altitude $18.4 \mathrm{~km}$ ). Simulations of $\beta$-NAT with $(\mathbf{a}, \mathbf{b}) \mathrm{AR}=0.1$ and $(\mathbf{c}, \mathbf{d}) \mathrm{AR}=10.0$ with size distributions A (red) and $\mathrm{B}$ (blue). Lower panels: residuals between the simulations and the observation. Black arrows denote prominent $\mathrm{H}_{2} \mathrm{O}$ absorption signatures from scattering of tropospheric radiation by PSC particles. Numbers in the legend refer to scenarios in Table 1. "NATcoa" corresponds to $\beta$-NAT, "TM" to T-matrix simulation, and "SP" to spheroid (numbers indicate AR).

and platelets with similar proportions depending on the crystallization conditions.

The results of the simulations with $\mathrm{AR}=0.1$ and $\mathrm{AR}=10.0$ are shown in Fig. 12. For $\mathrm{AR}=0.1$ and size distribution A, high systematic offsets of 100 to $200 \times$ $10^{-9} \mathrm{~W} \mathrm{~cm}^{-2} \mathrm{sr}^{-1} \mathrm{~cm}$ are found in channel 1 and up to $\sim$ $50 \times 10^{-9} \mathrm{~W} \mathrm{~cm}^{-2} \mathrm{sr}^{-1} \mathrm{~cm}$ in channel 2 (Fig. $12 \mathrm{a}$ and $\mathrm{b}$, red). In contrast, size distribution $B$ results in a much flatter residual pattern close to zero in wide ranges of channel 1 and in the entire channel 2 (Fig. 12a and b, blue). For both AR values and size distributions, the residual peak in the spectral region around $820 \mathrm{~cm}^{-1}$ flattens out to a high degree, in contrast to the previous simulations. The $\mathrm{H}_{2} \mathrm{O}_{(\mathrm{g})}$ absorption signatures in the spectral range from 1170 to $1250 \mathrm{~cm}^{-1}$ are reproduced well for size distribution $B$.

For $\mathrm{AR}=10.0$ and size distribution $\mathrm{A}$, high residual values of typically around 100 to $150 \times 10^{-9} \mathrm{~W} \mathrm{~cm}^{-2} \mathrm{sr}^{-1} \mathrm{~cm}$ are again found in channel 1 and mostly below $50 \times$ $10^{-9} \mathrm{~W} \mathrm{~cm}^{-2} \mathrm{sr}^{-1} \mathrm{~cm}$ in channel 2 (Fig. 12c and $\mathrm{d}$, red), whereas size distribution $\mathrm{B}$ results in improved agreement with the observation (Fig. 12c and d, blue). When compared to the corresponding $\mathrm{AR}=0.1$ scenario, increasingly negative residual values are found towards the upper boundary of channel 1, and slightly negative residual values below $1300 \mathrm{~cm}^{-1}$ in channel 2 . However, the spectral region around $820 \mathrm{~cm}^{-1}$ in channel 1 is reproduced slightly better than in the $\mathrm{AR}=0.1$ case.

Both scenarios, elongated spheroids with $\mathrm{AR}=0.1$ and oblate spheroids with $\mathrm{AR}=10.0$, result in considerably improved agreement with the observations when compared with the previous simulations. For both size distributions, the residual dip in the spectral region around $820 \mathrm{~cm}^{-1}$ flattens out significantly.

For size distribution $\mathrm{B}$, the spectral range above $900 \mathrm{~cm}^{-1}$ is furthermore reproduced well by the $\mathrm{AR}=0.1$ scenario, and good agreement is found in channel 2. As in the previous cases, residual patterns remain, especially in the densely populated spectral regions and at the channel boundaries. 


\subsection{Sensitivity to aspect ratio and comparison with refractive indices}

In the following, we compare the radiative transfer simulations of elongated spheroids consisting of $\beta$-NAT with different aspect ratios. The same comparison for oblate spheroids results in similar conclusions. Figure 13a and b show the results of the Mie scenario (AR $=1.0$, cyan) and the T-Matrix calculations with $\mathrm{AR}=0.5$ (magenta) and $\mathrm{AR}=0.1$ (green) for size distribution $\mathrm{B}$.

The scenarios with $\mathrm{AR}=1.0$ and 0.5 show only small differences of a few $10^{-9} \mathrm{~W} \mathrm{~cm}^{-2} \mathrm{sr}^{-1} \mathrm{~cm}$. For $\mathrm{AR}=0.5$, the residual peak in the spectral region around $820 \mathrm{~cm}^{-1}$ decreases slightly, and the spectrum is tilted slightly towards the observation in the higher wavenumber region of channel 1. Slightly improved agreement with the observation is also found in channel 2 . In contrast, the agreement with the observation is improved considerably for $\mathrm{AR}=0.1$ : in channel 1 , the residual dip in the spectral region around $820 \mathrm{~cm}^{-1}$ flattens out to a high degree. The simulated spectrum is tilted towards the observation above $900 \mathrm{~cm}^{-1}$ and reproduces the observed spectrum in this region well. In channel 2, higher radiances in close agreement with the observation are found when compared to the other scenarios.

Figure $13 \mathrm{c}$ and $\mathrm{d}$ show the imaginary parts of the refractive indices of $\beta$-NAT, $\alpha$-NAT, NAD, STS, and ice, which determine the absorption and emission characteristics of the particles. In the spectral region around $820 \mathrm{~cm}^{-1}$, which is weakly populated by trace gas signatures, $\beta$-NAT provides the strongest signature due to the $v_{2}$ symmetric deformation mode of $\mathrm{NO}_{3}^{-}$. Further broad peaks are found in channel 2 around $\sim 1330 \mathrm{~cm}^{-1}$ due to the $\nu_{3}$ asymmetric stretch mode of $\mathrm{NO}_{3}^{-}$and around $\sim 1200 \mathrm{~cm}^{-1}$ due to the $\nu_{2}$ symmetric umbrella mode of $\mathrm{H}_{3} \mathrm{O}^{+}$(Ortega et al., 2006, and references therein). These signatures however result in weak and broad residual patterns in the Mie scenarios, which are unspecific without further information (see Fig. 10). The $\alpha$ NAT refractive index imaginary part also shows a peak at $820 \mathrm{~cm}^{-1}$ due to the $\nu_{2}$ symmetric deformation mode of $\mathrm{NO}_{3}^{-}$of this NAT modification. The amplitude is however only about half of the amplitude of the used $\beta$-NAT refractive index, and it is superimposed by a broader signature beginning at $\sim 900 \mathrm{~cm}^{-1}$ and peaking at $\sim 780 \mathrm{~cm}^{-1}$. Similarly to $\alpha$-NAT, NAD also shows a peak at $808 \mathrm{~cm}^{-1}$ with a smaller amplitude when compared to $\beta$-NAT along with another peak with higher amplitude at $745 \mathrm{~cm}^{-1}$ in the region densely populated with trace gas signatures. Furthermore, NAD also shows broad peaks at $\sim 1260 \mathrm{~cm}^{-1}$ due to the $v_{3}$ mode $\mathrm{NO}_{3}^{-}$and at $\sim 1150 \mathrm{~cm}^{-1}$ (coinciding with the channel boundary) due to the $\nu_{2}$ mode of $\mathrm{H}_{3} \mathrm{O}^{+}$. For $\alpha$-NAT, only the increasing slopes of the corresponding signatures towards the boundaries of channel 2 can be identified. STS shows no significant peak-like signatures in both channels. For ice, a broad peak spanning the entire range of channel 1 is found due to librational modes in the ice crystal (Zasetsky et al., 2005).

Figure $13 \mathrm{e}$ and $\mathrm{f}$ show the real parts of the refractive indices of the discussed species, which determine the scattering of radiation from outside into the field-of-view. $\beta$ NAT shows the strongest "step-like" signature in the spectral region around $820 \mathrm{~cm}^{-1}$, corresponding to the peak in the refractive index imaginary part. $\alpha$-NAT and NAD again show weaker corresponding signatures at 820 and $808 \mathrm{~cm}^{-1}$. For NAD, another sharp step is seen at $\sim 745 \mathrm{~cm}^{-1}$ and a broad step around $\sim 1270 \mathrm{~cm}^{-1}$. Ice shows an extended decreasing gradient from the lower boundary of channel 1 to $\sim 930 \mathrm{~cm}^{-1}$. Neither ice nor STS shows any characteristic sharp signature in either channel.

The refractive indices of $\beta$-NAT show the strongest signature at $820 \mathrm{~cm}^{-1}$ in both the refractive index imaginary and real parts and allow us to model the "shoulder-like" signature around $820 \mathrm{~cm}^{-1}$ with a realistic spectral position and amplitude. $\alpha$-NAT and NAD also show similar but significantly weaker signatures at 820 and $808 \mathrm{~cm}^{-1}$, respectively. Therefore, potential highly aspherical large $\alpha$-NAT and NAD particles are expected to result in considerably weaker corresponding "shoulder-like" signatures along with further discrepancies from $\beta$-NAT as a consequence of different patterns in the refractive index imaginary and real parts.

Figure $13 \mathrm{~g}$ and $\mathrm{h}$ show the ensemble-averaged absorption and scattering cross sections of $\beta$-NAT for the considered size distribution for $\mathrm{AR}=0.1$ and $\mathrm{AR}=1.0$, which determine the absorption/emission and scattering characteristics of the simulated particles. The T-Matrix scenario with $\mathrm{AR}=0.1$ shows a much stronger peak in the absorption cross section and a stronger step in the scattering cross section in the spectral window around $820 \mathrm{~cm}^{-1}$ when compared to the Mie scenario, which together result in the characteristic "shoulder-like" signature in the simulated spectrum. Furthermore, the $\mathrm{AR}=0.1$ scenario shows considerably higher values of the scattering cross section towards higher wavenumbers, resulting in a relatively flat baseline of the simulated spectrum towards the upper end of channel 1 . In the $A R=0.1$ scenario, higher absorption and scattering cross sections in channel 2 result in higher radiances in the corresponding simulated spectrum.

The relative contributions of absorption/emission and scattering to the observed signature are investigated in Fig. 14 for the $\mathrm{AR}=1.0$ scenario (magenta) and the $\mathrm{AR}=0.1$ (green) scenario. Also shown are the same simulations neglecting the scattering by the particles (violet and dark green, respectively). Thereby, the wavenumber-dependent ensembleaveraged particle extinction coefficients are calculated taking into account only absorption and emission by the particles and neglecting the scattering source function.

For $\mathrm{AR}=0.1$, the scenario neglecting scattering by the particles results in lower radiances by up to $\sim 200 \times$ $10^{-9} \mathrm{~W} \mathrm{~cm}^{-2} \mathrm{sr}^{-1} \mathrm{~cm}$ in channel 1 (Fig. 14a) and $\sim 100 \times$ $10^{-9} \mathrm{~W} \mathrm{~cm}^{-2} \mathrm{sr}^{-1} \mathrm{~cm}$ in channel 2 (Fig. 14b) when com- 
(a)

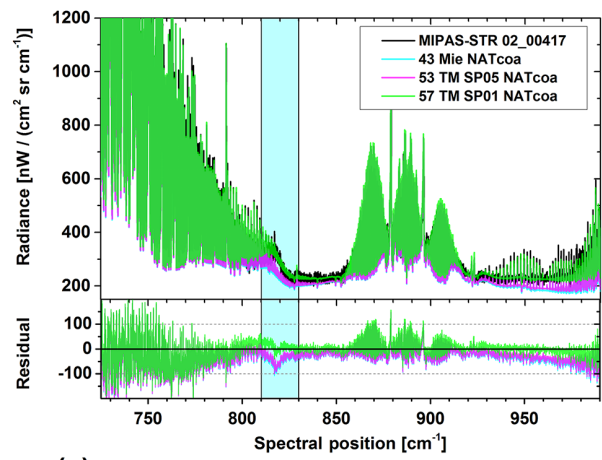

(c)

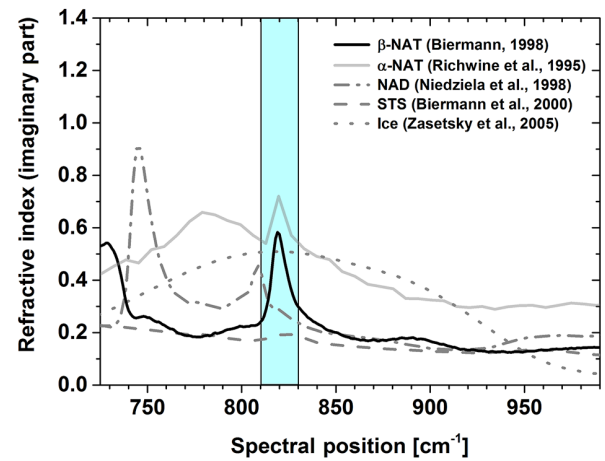

(e)

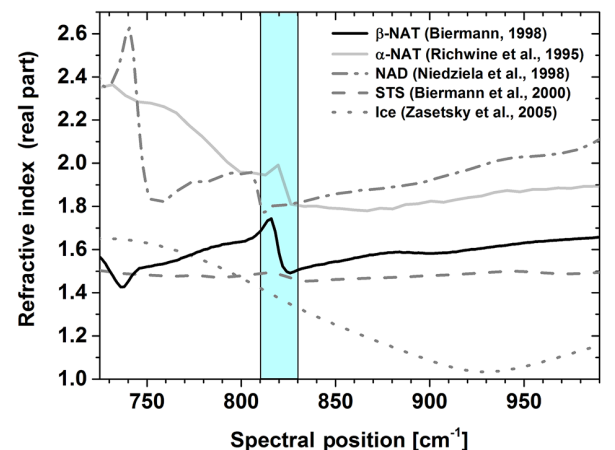

(g)

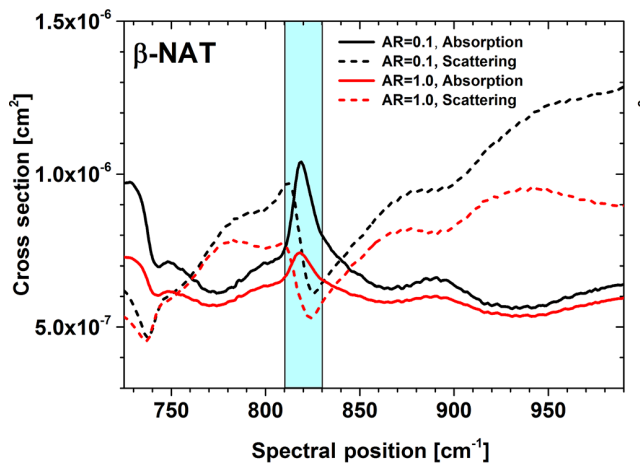

(b)

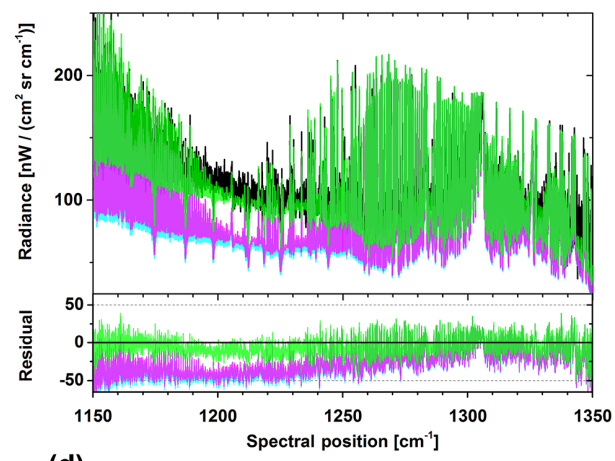

(d)

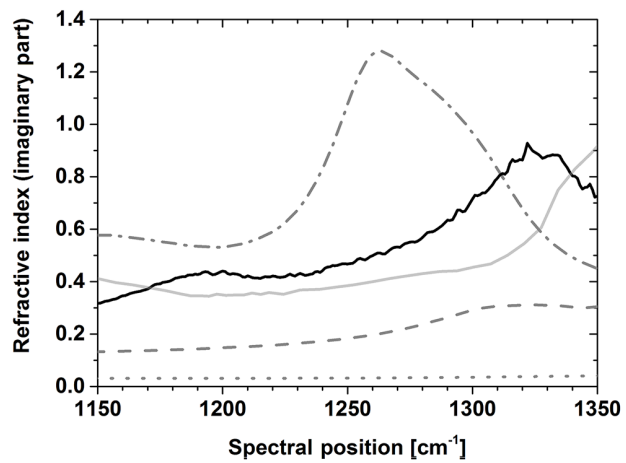

(f)

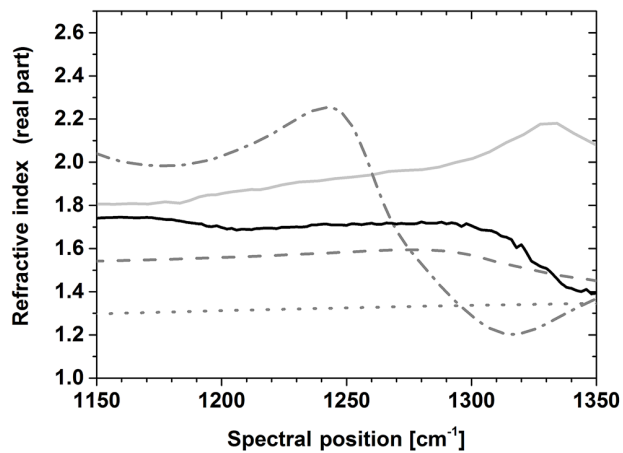

(h)

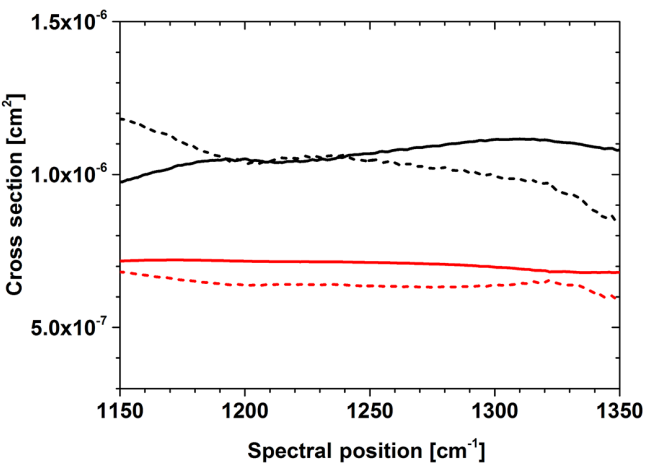

Figure 13. Dependence of simulated spectral signatures of $\beta$-NAT particles on the degree of non-sphericity. (a, b) Black: MIPAS-STR limb observation (13:47 UTC, tangent altitude $18.4 \mathrm{~km}$ ). Mie calculation for spherical particles (cyan), T-Matrix calculation with AR $=0.5$ (magenta), and T-Matrix calculation with $\mathrm{AR}=0.1$ (green). "NATcoa" corresponds to $\beta$-NAT, "Mie" to Mie simulation, "TM" to T-matrix simulation, and "SP" to spheroid (numbers indicate AR). Lower panels: residuals between the simulations and the observation. Numbers in the legend refer to scenarios in Table 1 . Refractive index $(\mathbf{c}, \mathbf{d})$ imaginary and (e, f) real parts of $\beta$-NAT, $\alpha$-NAT, NAD, STS, and ice. (g, h) Ensemble-averaged absorption and scattering cross sections of $\beta$-NAT for the discussed AR $=0.1$ and AR $=1.0$ scenarios. 
(a)

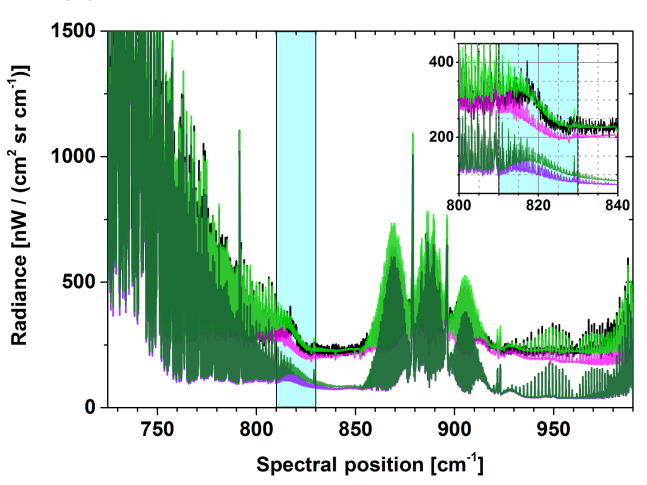

(b)

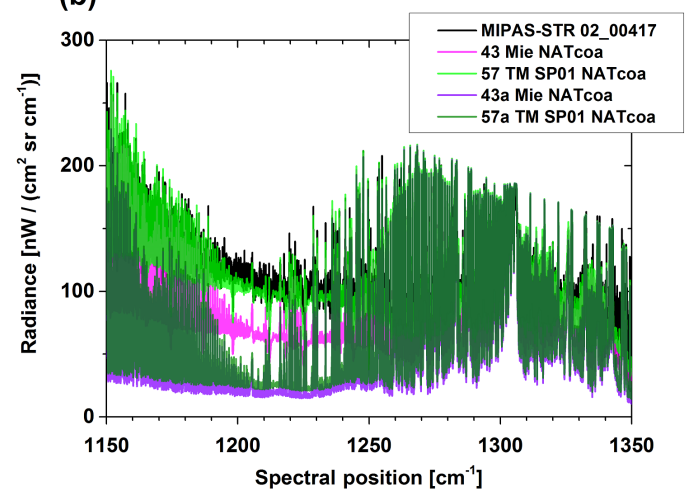

Figure 14. Relative roles of emission and scattering by spherical particles and highly elongated spheroids consisting of $\beta$-NAT. Black: MIPAS-STR limb observation (13:47 UTC, tangent altitude $18.4 \mathrm{~km})$. Magenta/violet: simulations of spherical particles $($ AR $=1.0)$ with/without scattering. Green/dark green: simulations of highly elongated particles $(A R=0.1)$ with/without scattering. Numbers in the legend refer to scenarios in Table 1. "NATcoa" corresponds to $\beta$-NAT, "Mie" to Mie simulation, "TM" to T-matrix simulation, and "SP" to spheroid (numbers indicate AR).

pared to the same scenario including scattering. This demonstrates the importance of the scattering source function. The same effect is found to a lesser degree also in the corresponding $\mathrm{AR}=1.0$ scenarios. The $\mathrm{AR}=1.0$ and $\mathrm{AR}=0.1$ scenarios neglecting scattering show only small differences on the order of $10 \times 10^{-9} \mathrm{~W} \mathrm{~cm}^{-2} \mathrm{sr}^{-1} \mathrm{~cm}$ from each other. Enhanced differences of $\sim 30 \times 10^{-9} \mathrm{~W} \mathrm{~cm}^{-2} \mathrm{sr}^{-1} \mathrm{~cm}$ are however found in the spectral region around $820 \mathrm{~cm}^{-1}$, where a weak "peak-like" signature with a maximum at $\sim 817 \mathrm{~cm}^{-1}$ can be identified in the $\mathrm{AR}=0.1$ scenario due to the net emission by the $820 \mathrm{~cm}^{-1}$ mode of $\beta$-NAT.

Much higher discrepancies between the $\mathrm{AR}=1.0$ and $\mathrm{AR}=0.1$ scenarios are seen if scattering is included, and a "shoulder-like" net signature peaking at $\sim 814 \mathrm{~cm}^{-1}$ is found in the $\mathrm{AR}=0.1$ scenario. In the observations, the shoulderlike signature is narrower than in the simulation and peaks at $\sim 818 \mathrm{~cm}^{-1}$. The simulation represents the increasing flank from higher towards lower radiances very well, while the maximum and the decreasing flank towards lower radiances are located at lower wavenumbers and are broader than in the simulation.

We point out that there are still remaining systematic discrepancies between the simulation of $\beta$-NAT for AR $=0.1$ and the observation. Besides the limitations of the radiative transfer simulation and scenario, the discrepancies are attributed to uncertainties in the refractive indices used $(30 \%$ uncertainty for imaginary parts; see Höpfner et al., 2006a; not quantified for real parts) and the fact that the refractive indices are only available at a spectral resolution of $1 \mathrm{~cm}^{-1}$. In contrast, the MIPAS-STR observations have a considerably higher apodized resolution of $0.069 \mathrm{~cm}^{-1}$. Another reason for the observed discrepancies might be the simplified particle shape assumed in the simulations. Real $\beta$-NAT particles might have different and more complex shapes and are likely to have edges and flat surfaces, altering their optical characteristics. This is supported by the experiments of Grothe et al. (2006), resulting in highly aspherical $\beta$-NAT particles (i.e., platelets and needles).

\subsection{Sensitivity calculations on the tropospheric scenario for $\beta$-NAT, AR $=0.1$}

In the following, we investigate the sensitivity of the $\mathrm{AR}=0.1$ scenario for $\beta$-NAT and size distribution $\mathrm{B}$ to modifications of the tropospheric cloud scenario and sea surface temperature. Figure 15 shows this scenario (Fig. 15ad, green, hereafter "optimized scenario") together with sensitivity simulations considering modified tropospheric cloud scenarios (see Table 1). The optimized scenario uses a tropospheric cloud layer between 0 and $10 \mathrm{~km}$ characterized by a continuum absorption coefficient of $0.0223 \mathrm{~km}^{-1}$ (at all wavelengths) and a sea surface temperature of $280.2 \mathrm{~K}$ (see Sect. 3.1).

The first modified tropospheric cloud scenario considering an opaque tropospheric cloud from 0 to $2 \mathrm{~km}$ and fully transparent conditions above hardly changes the simulated spectra in both channels when compared to the optimized scenario (Fig. 15a and b, magenta). This modified scenario is motivated by CALIPSO observations of tropospheric clouds during the scan from 11:23 to 11:26 UTC (not shown; see http: //www-calipso.larc.nasa.gov/products/), indicating a dense cloud layer below $\sim 2 \mathrm{~km}$. Note however that CALIPSO provides vertical cross sections corresponding to a narrow footprint and that the horizontal cloud distribution is variable. The second modified scenario neglects the presence of any tropospheric clouds (Fig. 15a and b, cyan). The comparison with the optimized scenario and the observation shows that the radiances in both channels are significantly overes- 
(a)

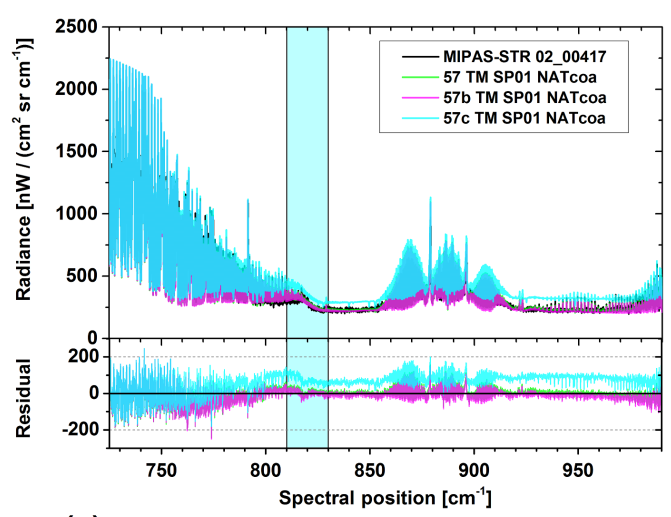

(c)

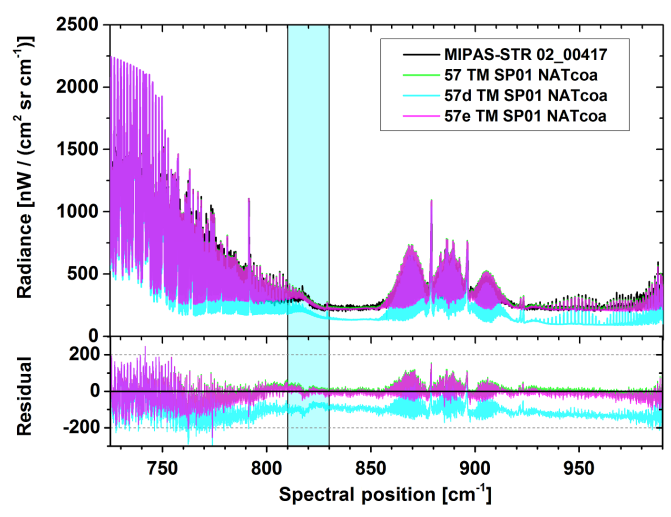

(b)

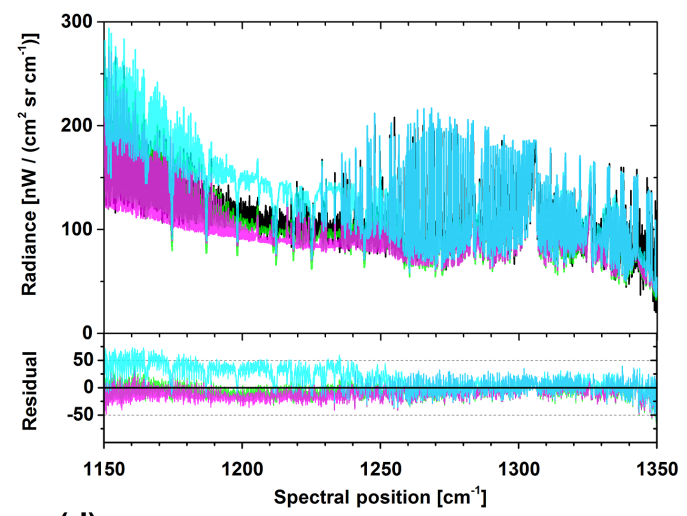

(d)

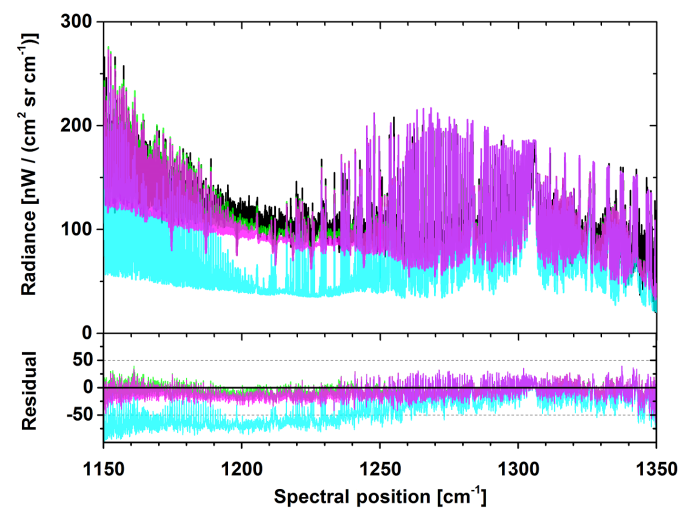

Figure 15. Influence of the tropospheric scenario on simulations of elongated spheroids with $\mathrm{AR}=0.1$ consisting of $\beta$-NAT. Black spectra in all panels: MIPAS-STR limb observation (13:47 UTC, tangent altitude $18.4 \mathrm{~km}$ ). Green spectra in all panels (mostly hidden by magenta spectra): optimized scenario. (a, b) Sensitivity simulations assuming an opaque tropospheric cloud between 0 and $2 \mathrm{~km}(\mathrm{magenta})$ and the absence of any tropospheric cloud (cyan). (c, d) Sensitivity simulations assuming an opaque tropospheric cloud from 0 to $10 \mathrm{~km}$ (cyan) and a surface temperature reduced by $7 \mathrm{~K}$ (magenta). Numbers in the legend refer to scenarios in Table 1. "NATcoa" corresponds to $\beta$-NAT, "TM" to T-matrix simulation, and "SP" to spheroid (numbers indicate AR).

timated. However, a "shoulder-like" signature in the spectral region around $820 \mathrm{~cm}^{-1}$ can be identified.

The third modified scenario considers the extreme case of an opaque tropospheric cloud between 0 and $10 \mathrm{~km}$ (Fig. 15c and d, cyan). High negative offsets are found in both channels when compared to the observation, and the $\mathrm{H}_{2} \mathrm{O}_{(\mathrm{g})}$ absorption signatures between 1170 and $1250 \mathrm{~cm}^{-1}$ are not reproduced. Accordingly, this scenario confirms the requirement for partially transparent conditions down to low tropospheric altitudes. However, this scenario also weakly indicates a "shoulder-like" signature around $820 \mathrm{~cm}^{-1}$. Finally, the scenario with a $7 \mathrm{~K}$ lower surface temperature (i.e., $273.2 \mathrm{~K}$, Fig. $15 \mathrm{c}$ and d, magenta) results only in small changes when compared to the optimized scenario. Accordingly, the identification of the "shoulder-like" signature around $820 \mathrm{~cm}^{-1}$ is robust against the adopted tropospheric cloud and tropospheric radiation emission scenario. The presence of partially transparent conditions down to the low troposphere is confirmed.

\subsection{Sensitivity calculations on the PSC scenario for $\beta$-NAT, AR $=0.1$}

In the following, the sensitivity of the simulated spectra to the thickness of the simulated PSC layer and the adopted particle size distribution is investigated. Figure 16 shows the optimized $\mathrm{AR}=0.1$ scenario (green) using size distribution $\mathrm{B}$ together with sensitivity simulations considering a reduced PSC top altitude and modified size distributions (see Fig. 9b and Tables 1 and 2). The scenario with a lower PSC top altitude of $20 \mathrm{~km}$ instead of $23 \mathrm{~km}$ shows a significant negative offset in both channels (Fig. 16a and b, orange). Both the signature around $820 \mathrm{~cm}^{-1}$ in channel 1 and the $\mathrm{H}_{2} \mathrm{O}_{(\mathrm{g})}$ absorption signatures in channel 2 show smaller amplitudes when compared to the optimized scenario.

The scenario considering only the second mode of the size distribution B (size distribution B1; see Fig. 9b and Table 2) results only in small changes when compared to the optimized scenario (Fig. 16a and b, cyan). Accordingly, the first 
(a)

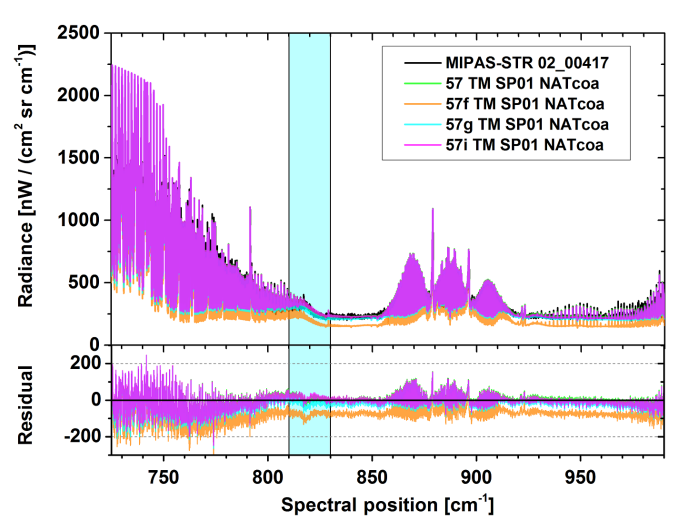

(b)

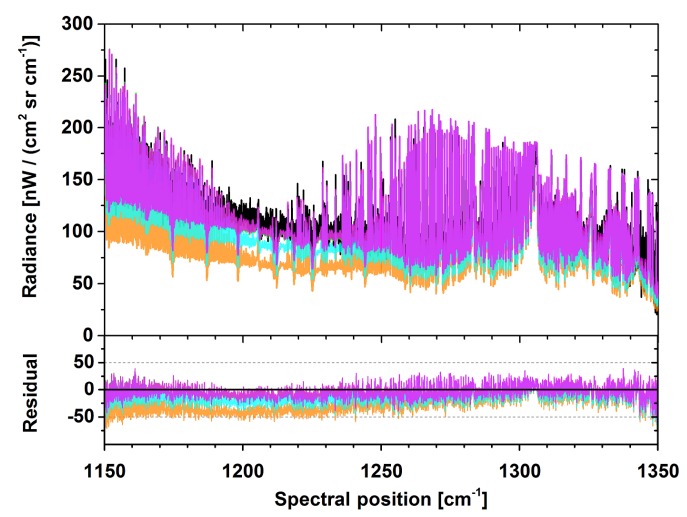

Figure 16. Influence of PSC layer thickness and particle size distribution on simulations of elongated spheroids with AR $=0.1$ consisting of $\beta$-NAT. Black: MIPAS-STR limb observation (13:47 UTC, tangent altitude $18.4 \mathrm{~km}$ ). Green (mostly hidden by magenta spectra): optimized scenario (assuming a vertical extent of the PSC from 17 to $23 \mathrm{~km}$ and size distribution B). Orange: sensitivity simulation assuming a reduced vertical extent of the PSC from 17 to $20 \mathrm{~km}$. Cyan and magenta: sensitivity simulations using size distributions B1 and B2. Numbers in the legend refer to scenarios in Table 1. For size distributions, see Table 2 and Fig. 9b. "NATcoa" corresponds to $\beta$-NAT, "TM" to T-matrix simulation, and "SP" to spheroid (numbers indicate AR).

mode of size distribution B is of minor importance for the simulated radiances. We furthermore investigate the role of a hypothetic mode of small $\beta$-NAT particles with a high number density, which might have been present above the flight path and contributed to the observed spectra. In size distribution B2 (see Fig. 9b and Table 2), the first mode is replaced by a small particle mode with a mode radius of $0.5 \mu \mathrm{m}$ and a number density enhanced by a factor of 100 . The corresponding total amount of condensed $\mathrm{HNO}_{3(\mathrm{~g})}$ is comparable with size distribution B (see Table 3). The resulting simulated spectra (Fig. 16a and b, magenta) are almost identical to the optimized scenario. Accordingly, the exact shape of the first mode is of minor importance, as long as the amount of condensed $\mathrm{HNO}_{3(\mathrm{~g})}$ is maintained.

So in summary, reducing the simulated PSC layer thickness by $50 \%$ results in significantly lower simulated radiances. However, the overall spectral pattern, a weak signature around $820 \mathrm{~cm}^{-1}$, and $\mathrm{H}_{2} \mathrm{O}_{(\mathrm{g})}$ absorption signatures between 1170 and $1250 \mathrm{~cm}^{-1}$ can be identified. Omitting the first mode of particle size distribution B or replacing the first mode by a small particle mode with similar $\mathrm{HNO}_{3}$ content results only in small changes in the simulated spectra. Accordingly, the identification of the $\beta$-NAT signature around $820 \mathrm{~cm}^{-1}$ is robust against the assumed thickness of the PSC layer and modifications of the size distribution in the considered ranges. The second mode of size distribution $\mathrm{B}$ with a mode radius of $4.8 \mu \mathrm{m}$ plays the key role in the simulation of the overall offset due to scattering and the "shoulder-like" signature around $820 \mathrm{~cm}^{-1}$.

We furthermore perform a sensitivity study based on the scenario involving the simplified size distribution B1 to investigate the effect of decreasing mode radii on the observed spectral signatures when the total volume of $\beta$-NAT is kept constant. The results are reported in Appendix B and show that the transition from a "shoulder-like" to a "peak-like" signature occurs for $\mathrm{AR}=0.1$ and the considered mode width at a mode radius of $\sim 3.0 \mu \mathrm{m}$. For a mode radius of $1.0 \mu \mathrm{m}$, a "peak-like" signature is found in agreement with a corresponding Mie simulation. The results show furthermore that a modified "shoulder-like" signature along with further changes in the simulated spectra result for spherical particles with a mode radius of $3.0 \mu \mathrm{m}$.

\section{Comparison of simulated and observed particle size distributions}

In the following, we compare the amounts of gas-phase equivalent $\mathrm{HNO}_{3}$ corresponding to size distributions A, B, $\mathrm{B} 1$, and $\mathrm{B} 2$ with the same quantity derived from the in situ observations by the FSSP-100 and CDP (see Tables 2 and 3 and Fig. 9). Gas-phase equivalent $\mathrm{HNO}_{3}$ is calculated for pressure and temperature at flight altitude and corresponds to the volume mixing ratio of gaseous $\mathrm{HNO}_{3}$ added to the gas phase if the particles would evaporate instantaneously. The results are furthermore compared with a corresponding size distribution simulated by the CLaMS for the area from 70.0 to $72.0^{\circ} \mathrm{N}$ and 15.0 to $22.5^{\circ} \mathrm{E}$ in the altitude range from $\sim 18.0$ to $18.5 \mathrm{~km}$ at 14:00 UTC (Fig. 9b). Finally, the results are compared with excess $\mathrm{HNO}_{3(\mathrm{~g})}$ in the nitrification layer below the flight path retrieved from the MIPAS-STR measurements.

The bimodal size distribution A corresponds to $18.2 \mathrm{ppbv}$ gas-phase equivalent $\mathrm{HNO}_{3}$ and resembles approximately the size distribution derived from the FSSP-100 observa- 
Table 3. Comparison of equivalent $\mathrm{HNO}_{3(\mathrm{~g})}$ corresponding to the particle size distributions used in the radiative transfer simulations of the MIPAS-STR observations, the size distributions derived from the in situ measurements, and a CLaMS simulation, together with excess $\mathrm{HNO}_{3(\mathrm{~g})}$ derived for the nitrification layer below the observed PSC.

\begin{tabular}{lrl}
\hline Size distribution & $\mathrm{HNO}_{3(\mathrm{~g})}(\mathrm{ppbv})$ & Comment \\
\hline $\mathrm{A}$ & 18.2 & Radiative transfer simulation, starting point \\
$\mathrm{B}$ & 9.3 & Radiative transfer simulation, optimized scenario \\
$\mathrm{B} 1$ & 8.4 & Radiative transfer simulation, sensitivity \\
$\mathrm{B} 2$ & 9.8 & Radiative transfer simulation, sensitivity \\
FSSP-100 & 18.5 & Derived from in situ observation \\
CDP & 28.5 & Derived from in situ observation \\
CLaMS & 1.6 & Chemistry transport simulation \\
Nitrification layer* & 5.0 & Excess $\mathrm{HNO}_{3(\mathrm{~g})} @ \sim 16.0 \mathrm{~km}$ \\
& 7.5 & Equivalent $\mathrm{HNO}_{3(\mathrm{~g})} @ 18.5 \mathrm{~km}$ \\
\hline
\end{tabular}

\footnotetext{
* See Sect. 2.5 .
}

tions, which corresponds to $18.5 \mathrm{ppbv}$ of gas-phase equivalent $\mathrm{HNO}_{3}$. The optimized size distribution $\mathrm{B}$ and size distributions $\mathrm{B} 1$ and $\mathrm{B} 2$ correspond to gas-phase equivalent $\mathrm{HNO}_{3}$ from 8.4 to 9.8 ppbv. Size distribution B was derived from size distribution A by reducing the mode radii of both modes by $20 \%$. The corresponding large difference in condensed $\mathrm{HNO}_{3}$ is the consequence of the high sensitivity of particle volume to particle radius.

The CLaMS size distribution shown in Fig. 9b includes an STS mode (below $3 \mu \mathrm{m}$ in the shown range of $\mathrm{d} N / \mathrm{d} \log (D)$ ) and a binned NAT mode. While the small STS mode hardly contributes to the total amount of condensed $\mathrm{HNO}_{3}$ in the simulation, the NAT mode contains a considerably smaller amount of gas-phase equivalent $\mathrm{HNO}_{3}$ of only $\sim 1.6 \mathrm{ppbv}$ when compared to size distribution $\mathrm{B}$ and more than 1 order of magnitude less gas-phase equivalent $\mathrm{HNO}_{3}$ than derived from the in situ observations. In the CLaMS simulation, a narrower NAT mode results, peaking at the size bin centered at $r=3.7 \mu \mathrm{m}$. The largest particles are found in the size bin centered at $r=4.7 \mu \mathrm{m}$.

While the occurrence of PSCs and the overall denitrification are reproduced well by CLaMS in general (Grooß et al., 2014), the discrepancy between the size distribution simulated by CLaMS and derived from the observations discussed here might by linked to uncertainties in the simulated NAT nucleation rates, missing nucleation processes (e.g., NAT nucleation on pre-existing ice), and simulated particle growth rates.

Furthermore, sedimentation rates for spherical particles are assumed in the CLaMS simulations. However, aspherical particles, such as supported by the observations discussed here, would have lower sedimentation rates than spherical NAT particles, which affects the time-dependent development of particle size distributions inside $\mathrm{PSCs}$ and $\mathrm{HNO}_{3}$ redistribution in general (Woiwode et al., 2014). Furthermore, locations and altitudes of specific PSCs and atmospheric structures in model simulations and observations can differ from each other, while the overall processes are represented in a realistic way in the model simulations.

The maximum amount of excess $\mathrm{HNO}_{3(\mathrm{~g})}$ of $\sim 5 \mathrm{ppbv}$ in the nitrification layer below the flight track (i.e., exceeding the extra-vortex correlation in Fig. 8) is used to put the amounts of condensed $\mathrm{HNO}_{3}$ (s) corresponding to the different size distributions into perspective. $\mathrm{HNO}_{3(\mathrm{~s})}$ at flight altitude corresponding to the size distributions also represents excess $\mathrm{HNO}_{3}$ originating from higher altitudes, as (i) the $\mathrm{O}_{3}-\mathrm{HNO}_{3(\mathrm{~g})}$ correlation at flight altitude already matches or slightly exceeds the extra-vortex correlation and (ii) the large particle sizes derived from the observations imply significant sedimentation rates and therefore an origin from higher altitudes. Converting the amount of excess $\mathrm{HNO}_{3}$ present at $\sim 16 \mathrm{~km}$ in terms of molecules per volume to the pressure at the flight altitude of $\sim 18.5 \mathrm{~km}$ results in a mixing ratio of $\sim 7.5$ ppbv, which is comparable with gas-phase equivalent $\mathrm{HNO}_{3}$ derived from size distributions B, B1, and B2. However, this comparison has to be made with care, since the excess $\mathrm{HNO}_{3}(\mathrm{~g})$ in the nitrification layer might have resulted from gradual evaporation of large particles settling into layers with temperatures above $T_{\text {NAT }}$ rather than simultaneous evaporation of a certain PSC layer due to rising temperatures. The spatial coincidence of the PSC and the nitrification layer below, the observations of large particles, and the fact that temperatures exceed $T_{\mathrm{NAT}}$ below the flight path strongly suggest that an ongoing denitrification process was observed and that the nitrification layer below the flight path was associated with the PSC above.

Accordingly, the size distributions B, B1, and B2 supported by the simulations of the MIPAS-STR spectra suggest by factors of $\sim 2$ to 3 smaller amounts of condensed $\mathrm{HNO}_{3}$ when compared to the size distributions derived from the in situ observations. We mention that Borrmann et al. (2000) investigated the effects of spheroids with $\mathrm{AR}=0.5$ on FSSP observations. Similar to the infrared observations discussed here, the results were close to corresponding Mie calcula- 
tions. However, the effects of highly aspherical particles on the interpretation of FSSP measurements are uncertain and might explain this discrepancy.

The larger particle sizes derived from the FSSP-100 and CDP measurements using Mie theory are not necessarily in contradiction with the radiative transfer simulations of the MIPAS-STR observations discussed here when interpreted as maximum dimensions of highly aspherical particles. For example, elongated spheroids with extreme aspect ratios can easily span lengths of several tens of microns while having relatively small individual particle volumes. Evidence of particles with sizes of this magnitude is provided by CIP shadow cast images recorded during the Arctic winter 2009/10 (Molleker et al., 2014).

The CLaMS simulation suggests a smaller amount of condensed $\mathrm{HNO}_{3}$ by a factor of $\sim 6$ when compared with size distribution $\mathrm{B}$ and by more than 1 order of magnitude less condensed $\mathrm{HNO}_{3}$ when compared with the size distributions derived from the in situ observations. The amount of excess $\mathrm{HNO}_{3(\mathrm{~g})}$ in the nitrification layer below the flight path is of the same magnitude as $\mathrm{HNO}_{3(\mathrm{~s})}$ corresponding to size distributions B, B1, and B2.

\section{Conclusions}

The MIPAS-STR observations associated with the Arctic PSC flight on 11 December 2011 show a characteristic "shoulder-like" signature in the spectral region around $820 \mathrm{~cm}^{-1}$, which is attributed to the $v_{2}$ symmetric deformation mode of $\mathrm{NO}_{3}^{-}$of $\beta$-NAT. The observed signature is explained by the absorption/emission and scattering characteristics of large highly aspheric $\beta$-NAT particles. While Mie calculations and T-Matrix calculations assuming spheroids with moderate AR values of 0.5 and 2.0 do not reproduce the observed signature and the overall spectral patterns, TMatrix calculations for spheroids involving extreme AR values of 0.1 and 10.0 result in reasonable agreement with the observations. In the discussed scenario, best agreement is found for $\mathrm{AR}=0.1$ and a bimodal lognormal size distribution with mode radii of 2.0 and $4.8 \mu \mathrm{m}$ when adopting a particle size distribution similar in shape with the results from the collocated in situ observations. The smaller mode with a mode radius of $2.0 \mu \mathrm{m}$ plays only a minor role in the simulations. The fact that best agreement is found for highly elongated particles might indicate a heterogeneous nucleation of the particles involving ice and a subsequent phase transition from $\alpha$-NAT to $\beta$-NAT (Grothe et al., 2006; Iannarelli and Rossi, 2016; Weiss et al., 2016, and references therein). While the temperatures at flight altitude were too warm for ice and $\alpha$-NAT and model temperatures do not support a previous ice nucleation, Molleker et al. (2014) suggest that ice particles might have nucleated previously during lee-waveinduced cooling above Greenland not resolved by the model, enabling an ice-induced nucleation of NAT.
The $\nu_{2}$ symmetric deformation mode of $\mathrm{NO}_{3}^{-}$at $820 \mathrm{~cm}^{-1}$ is well suited for identification of $\beta$-NAT, since it is located in a spectral region weakly populated by gaseous absorbers and where the thermal emission of the atmosphere is higher than at higher wavenumbers. Furthermore, it represents a sharp feature, having a significant amplitude in both the refractive index imaginary and real parts. The identification of large aspherical $\beta$-NAT particles is furthermore supported by the reasonable overall agreement of the simulations with the observations in the entire spectral regions analyzed, covering further weak and broad signals due to the $v_{3}$ asymmetric stretch mode of $\mathrm{NO}_{3}^{-}$around $\sim 1330 \mathrm{~cm}^{-1}$, the $\nu_{2}$ symmetric umbrella mode of $\mathrm{H}_{3} \mathrm{O}^{+}$at $\sim 1200 \mathrm{~cm}^{-1}$, and unspecified patterns. The combination of these signatures results in a specific fingerprint in the absorption and scattering cross sections of highly aspherical large $\beta$-NAT particles. Potential highly aspherical large $\alpha$-NAT and $\alpha$-NAD particles are expected to result in considerably weaker "shoulder-like" signatures at $\sim 820 \mathrm{~cm}^{-1}$ and $\sim 808 \mathrm{~cm}^{-1}$ due to weaker corresponding signatures in the refractive indices along with further discrepancies from $\beta$-NAT due to different patterns in the refractive indices at higher wavenumbers.

Sensitivity calculations involving a simplified size distribution show that for $\mathrm{AR}=0.1$ the transition from a "shoulder-like" to a "peak-like" signature occurs at a mode radius of $\sim 3.0 \mu \mathrm{m}$. A developed "peak-like" signature as discussed by Höpfner et al. (2006a) is found for a mode radius of $1.0 \mu \mathrm{m}$, which is almost identical to the corresponding Mie simulation. Furthermore, a corresponding Mie simulation with a mode radius of $3.0 \mu \mathrm{m}$ shows that a modified "shoulder-like" signature along with further changes in the modeled spectra can be simulated for spherical particles using the discussed size distribution.

The combination of the MIPAS-STR cloud, temperature, and trace gas data products, collocated in situ observations aboard the Geophysica, CALIPSO observations, and radiosonde observations allows us to define the radiative transfer scenario accurately. The scattering of upwelling tropospheric radiation by the particles plays an important role in the simulation of the overall spectral patterns and in particular of the "shoulder-like" signature around $820 \mathrm{~cm}^{-1}$. The importance of scattering is also reflected by the observation of prominent $\mathrm{H}_{2} \mathrm{O}_{(\mathrm{g})}$ absorption signatures in the MIPAS-STR limb emission spectra between 1170 and $1250 \mathrm{~cm}^{-1}$, implying scattering of radiation from the low troposphere/surface into the field-of-view. Remaining discrepancies between the radiative transfer simulations and the observations are attributed to the limitations of the radiative transfer simulation and scenario, the uncertainties and the comparably coarse spectral resolution of the available refractive index data, and the uncertain particle geometries. Further combinations of particle shapes (e.g., including edges and flat surfaces), aspect ratios, and potential shape-dependent 
orientation effects might further improve the agreement of simulations and observations.

The radiative transfer simulations of the MIPAS-STR observations suggest $\sim 20 \%$ smaller radii when compared to the size distributions derived from the in situ observations, resulting in factors of 2-3 smaller amounts of condensed $\mathrm{HNO}_{3}$. A corresponding CLaMS simulation suggests a factor of $\sim 6$ less condensed $\mathrm{HNO}_{3}$ and smaller particles when compared to the radiative transfer simulations. This discrepancy might be linked to uncertainties and limitations of the NAT nucleation, particle growth, and particle sedimentation rates (which would be different for highly aspherical particles) simulated by CLaMS and the limited comparison with the localized case study being discussed. Excess $\mathrm{HNO}_{3(\mathrm{~g})}$ derived from the MIPAS-STR observations in the nitrification layer below the observed PSC is of the same magnitude as $\mathrm{HNO}_{3 \text { (s) }}$ at a flight altitude corresponding to the size distribution supported by the radiative transfer simulations.

The discrepancies between the particle size distributions derived from the MIPAS-STR observations and the in situ observations might be due to the fact that spherical particles were assumed in the evaluation of the in situ observations. On the other hand, the particle sizes derived from the in situ observations may be reconciled with the simulations of the MIPAS-STR observations when interpreted as the maximum dimensions of highly aspherical particles. The results of our radiative transfer calculations suggest smaller volumes of individual particles than suggested by non-optical measurements during other PSC flights (Fahey et al., 2001; Molleker et al., 2014). However, the particle sizes derived here suggest significant sedimentation rates and associated vertical redistribution of $\mathrm{HNO}_{3}$. We mention that the shapes of particle size distributions of PSCs depend on the specific growing conditions of the particles. Furthermore, there might be some diversity in the NAT phase in terms of particle sizes and shapes, which is not captured by the assumption of lognormal size distributions and a single particle geometry. Real stratospheric $\beta$-NAT particles might have more complex shapes similar to ice (e.g., Libbrecht, 2005), and hollow NAT shells were proposed as an alternative explanation for large particle sizes derived from in situ measurements (Molleker et al., 2014; see also Biermann et al., 1998).
The shapes and sizes of large $\beta$-NAT particles are important parameters for simulations of denitrification, chemistry, and radiation in the polar stratosphere and for the interpretation of infrared limb observations, in situ particle measurements, and lidar observations. The presented results show that large highly aspherical $\beta$-NAT particles show a characteristic spectral signature, which may be exploited for the identification of large $\beta$-NAT particles involved in denitrification. Further laboratory measurements of the refractive indices of $\beta$-NAT particles with high spectral resolution would help to improve radiative transfer simulations and retrievals from high-resolution mid-infrared limb observations. The application of the T-Matrix method for highly aspherical particle shapes would help in the interpretation of in situ forward scattering measurements of $\beta$-NAT particles (compare Borrmann et al., 2000). Further airborne remote sensing and in situ PSC observations in the Arctic stratosphere using the combination of optical and non-optical methods would help to better characterize $\beta$-NAT particles, and advanced in situ measurements would allow actual measurement of the particle shapes (e.g., Kaye et al., 2008).

\section{Data availability}

Contact for accessing the MIPAS-STR data used in this study is provided at the website of the Remote Sensing using Aircraft and Balloons group of the Institute of Meteorology and Climate Research at the Karlsruhe Institute of Technology (see http://www.imk-asf.kit.edu/english/ffb.php). CALIPSO data was provided by the CALIPSO Science Team (2015) at doi:10.5067/CALIOP/CALIPSO/CAL_LID_L2_PSCMaskProv-V1-00_L2-001.00. Contact for accessing the FSSP100, CDP and FISH data is provided at the ESSenCe website (https://www.essence11.org/Essence). MIPAS-ENVISAT spectra can be accessed via https://earth.esa.int/web/guest/ pi-community/apply-for-data/fast-registration. Contact for accessing the CLaMS simulation used in this study is provided at the website of the Institute of Energy and Climate Research (IEK-7) at the Forschungszentrum Jülich GmbH (http://www.fz-juelich.de/iek/iek-7/EN/UeberUns/ Ansprechpartner/_node.html). 


\section{Appendix A}

The vertical cross sections of $\mathrm{H}_{2} \mathrm{O}_{(\mathrm{g})}$ and $\mathrm{O}_{3}$ retrieved from the MIPAS-STR observations are shown in Fig. A1. In the $\mathrm{O}_{3}$ cross section (Fig. A1b), the mixing ratios around and below flight altitude decrease in section $\mathrm{b}$ and increase again at the beginning of section c, similar to $\mathrm{HNO}_{3(\mathrm{~g})}$ (see Fig. 6b), and indicate the crossing of the vortex edge (see Fig. 1).

The absence of a local $\mathrm{O}_{3}$ maximum between 13:15 and 14:15 UTC around $\sim 16 \mathrm{~km}$ confirms that the $\mathrm{HNO}_{3(\mathrm{~g})}$ maximum in Fig. $6 \mathrm{~b}$ is the result of a nitrification process. The vertical profiles of $\mathrm{HNO}_{3(\mathrm{~g})}, \mathrm{H}_{2} \mathrm{O}_{(\mathrm{g})}$, and $\mathrm{O}_{3}$ used for the calculation of $T_{\mathrm{NAT}}$ and the $\mathrm{O}_{3}-\mathrm{HNO}_{3(\mathrm{~g})}$ correlation in Sect. 2.5 are shown in Fig. A2.

(a)

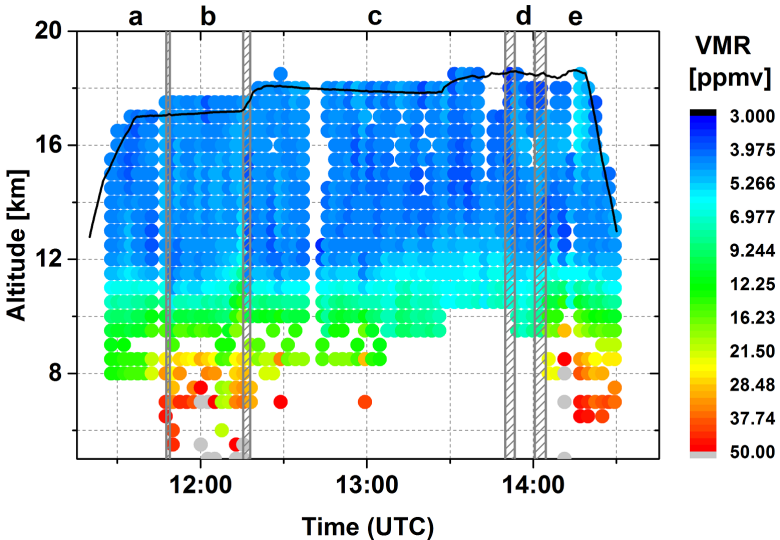

(b)

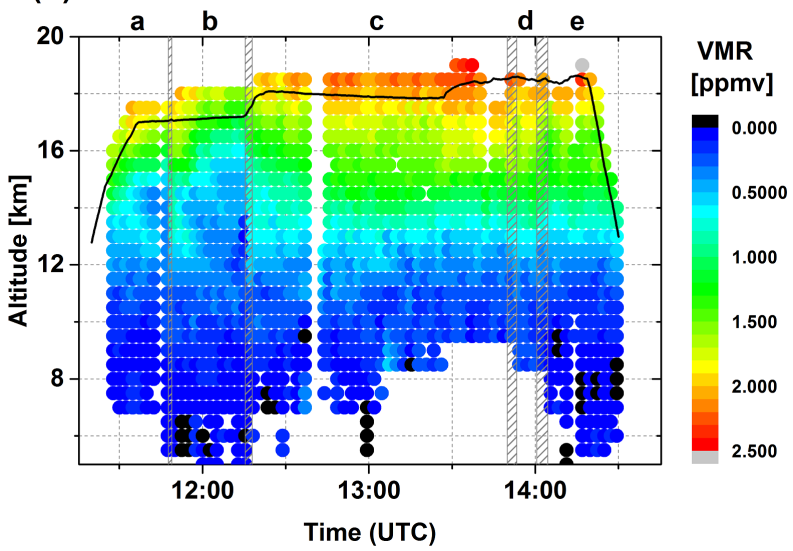

Figure A1. Vertical cross sections of (a) $\mathrm{H}_{2} \mathrm{O}_{(\mathrm{g})}$ and (b) $\mathrm{O}_{3}$ along flight track retrieved from the MIPAS-STR observations. Vertical flight profile (black line) and turns between different flight legs (grey hatched areas). Data points are filtered for a vertical resolution better than $5 \mathrm{~km}$. (a)

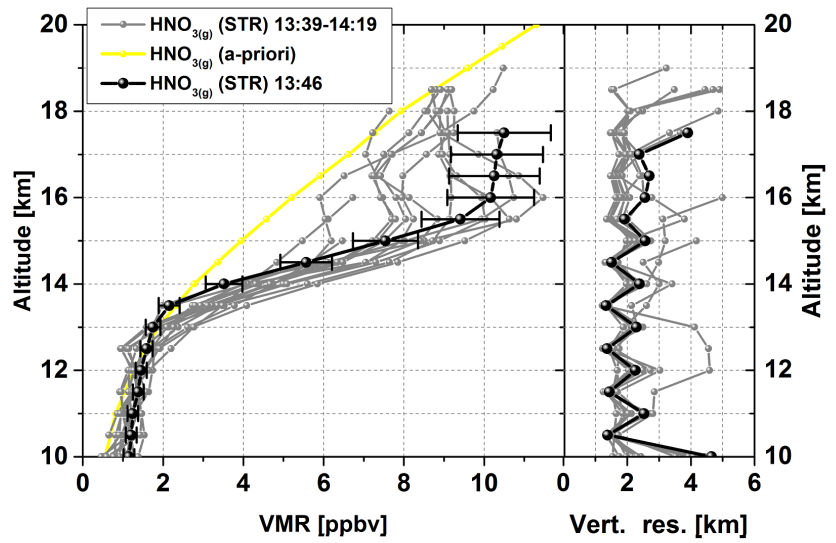

(b)

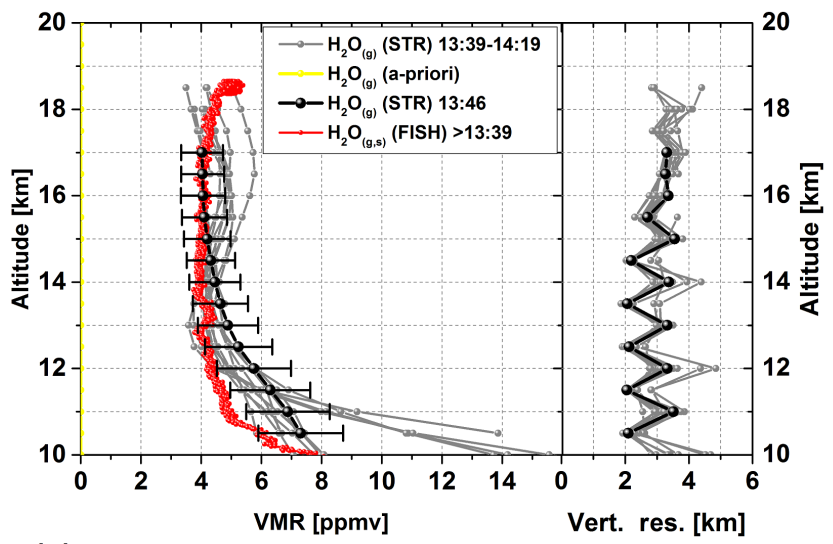

(c)

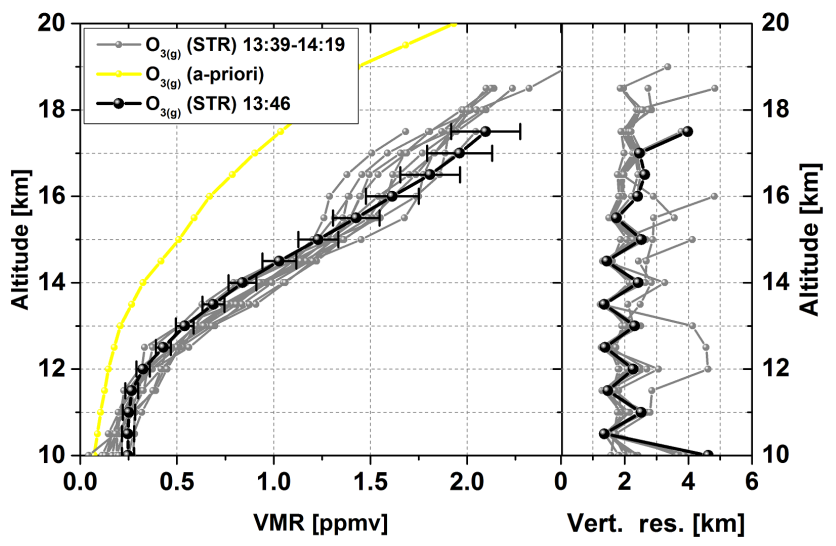

Figure A2. Left panels: vertical profiles of $\mathrm{HNO}_{3(\mathrm{~g})}, \mathrm{H}_{2} \mathrm{O}_{(\mathrm{g})}$, and $\mathrm{O}_{3}$ retrieved from the MIPAS-STR observations during the PSC encounter (grey and black) including initial guess/a priori profiles (yellow; $\mathrm{HNO}_{3(\mathrm{~g})}$ and $\mathrm{O}_{3}$ : Remedios et al., 2007; $\mathrm{H}_{2} \mathrm{O}_{(\mathrm{g})}$ : 0 ppmv at all altitudes). For $\mathrm{H}_{2} \mathrm{O}$, collocated FISH total $\mathrm{H}_{2} \mathrm{O}_{(\mathrm{g}+\mathrm{s})}$ measurements during the PSC encounter and the subsequent descent phase are shown for comparison. Right panels: vertical resolutions of MIPAS-STR profiles. 


\section{Appendix B}

The goal of the sensitivity study discussed in the following is to identify an approximate size threshold for particles with $\mathrm{AR}=0.1$ for the transition from a "peak-like" (compare Höpfner et al., 2006a) to a "shoulder-like" signature in the spectral region around $820 \mathrm{~cm}^{-1}$. Corresponding Mie calculations for spherical particles ( $\mathrm{AR}=1.0$ ) are shown for comparison. The starting point for the simulations is the simplified size distribution B1 (1-modal, $r=4.8 \mu \mathrm{m}$; see Fig. 9a and Table 1 , scenario $57 \mathrm{~g}$ ). Sensitivity calculations involve the same total volume of $\beta$-NAT (i.e., condensed $\mathrm{HNO}_{3}$ ) and mode radii of 3.0 and $1.0 \mu \mathrm{m}$, respectively (Fig. B1).

The results show that for $A R=0.1$ the spectral signature around $820 \mathrm{~cm}^{-1}$ becomes increasingly "peak-like" for mode radii decreasing from 4.8 to $1.0 \mu \mathrm{m}$ (Fig. B2a, c, and $\mathrm{e}$, blue). While for $r=4.8 \mu \mathrm{m}$ the signature shows a characteristic "shoulder-like" pattern, a superposition of "shoulderlike" and "peak-like" signatures results for $r=3.0 \mu \mathrm{m}$. A developed "peak-like" signature as discussed by Höpfner et al. (2006a) is found for $r=1.0 \mu \mathrm{m}$, and the simulated spectra are almost identical to the corresponding Mie scenario for both channels (Fig. B2e and f) except for slightly higher radiances below $\sim 860 \mathrm{~cm}^{-1}$ in for the $\mathrm{AR}=0.1$ scenario. Finally, the Mie calculations show that a modified "shoulderlike" signature around $820 \mathrm{~cm}^{-1}$ along with further differences from the $\mathrm{AR}=0.1$ scenario can be modeled for spherical particles with $r=3.0 \mu \mathrm{m}$.

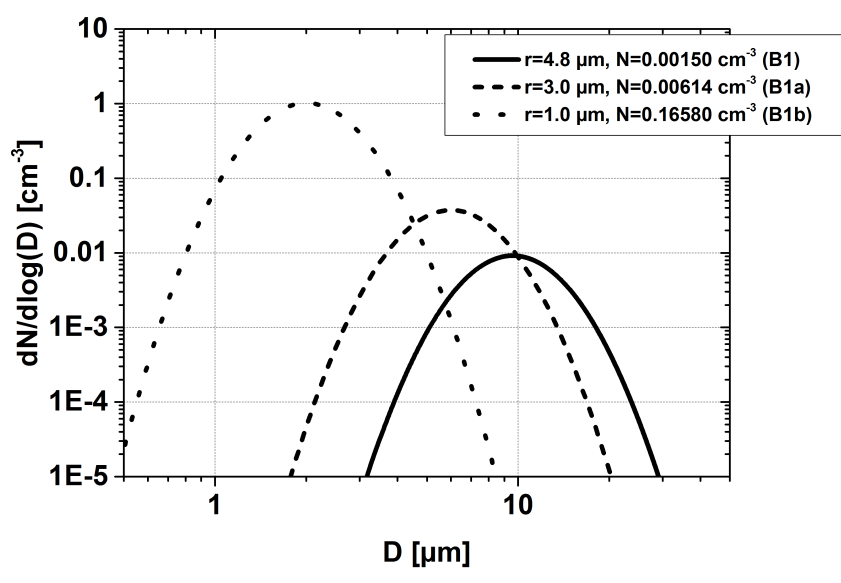

Figure B1. Size distributions used for sensitivity simulations discussed in Appendix B. The total volume of condensed $\beta$-NAT is constant (corresponding to 8.4 ppbv of gas-phase equivalent $\mathrm{HNO}_{3}$ ) and the mode width is 1.35 in all cases. For the binned size distribution used in the $\mathrm{AR}=0.1$ scenario with $r=1.0$, the particle number density had to be scaled to $0.17342 \mathrm{~cm}^{-3}$ to match $8.4 \mathrm{ppbv}$ of gasphase equivalent $\mathrm{HNO}_{3}$. 
(a)

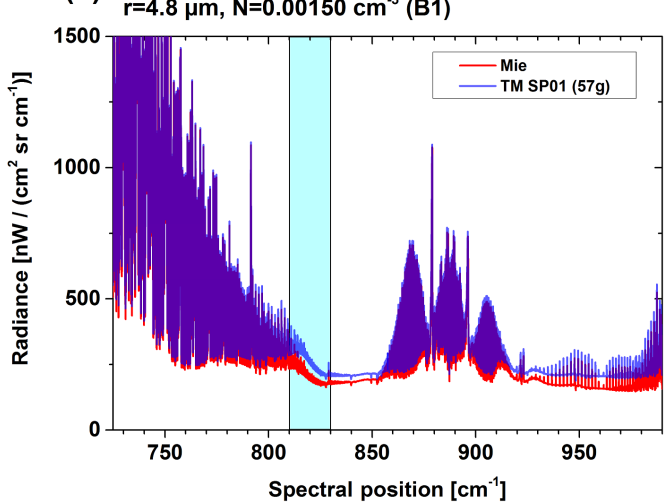

(c)

$r=3.0 \mu \mathrm{m}, \mathrm{N}=0.00614 \mathrm{~cm}^{-3}(\mathrm{~B} 1 \mathrm{a})$

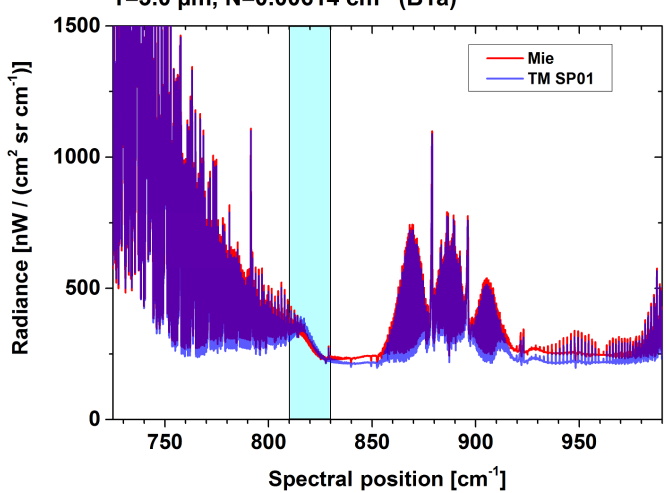

(e)

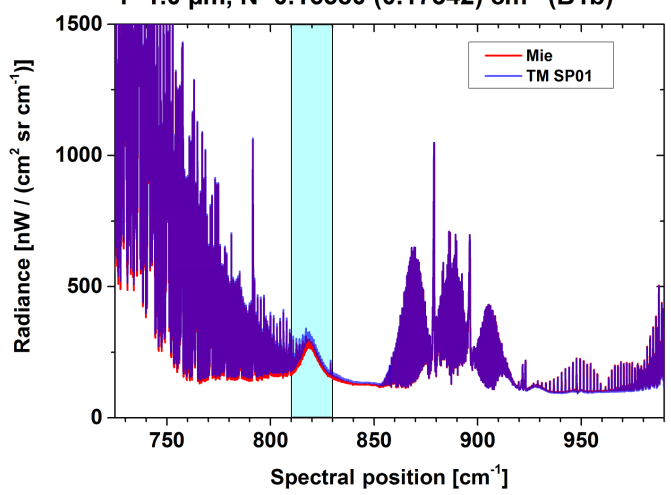

(b)

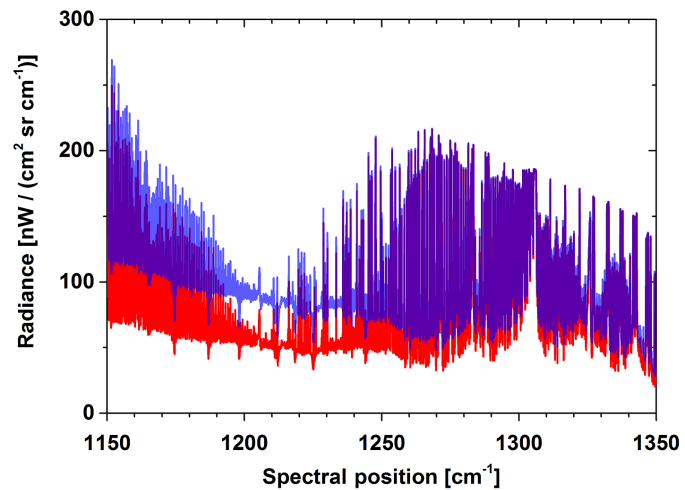

(d)

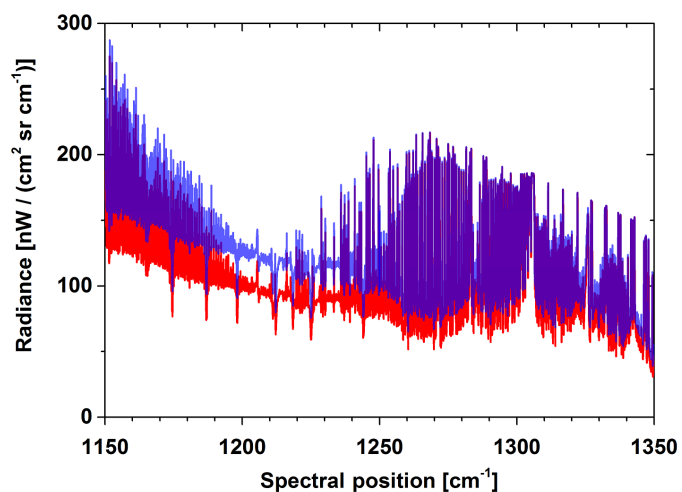

(f)

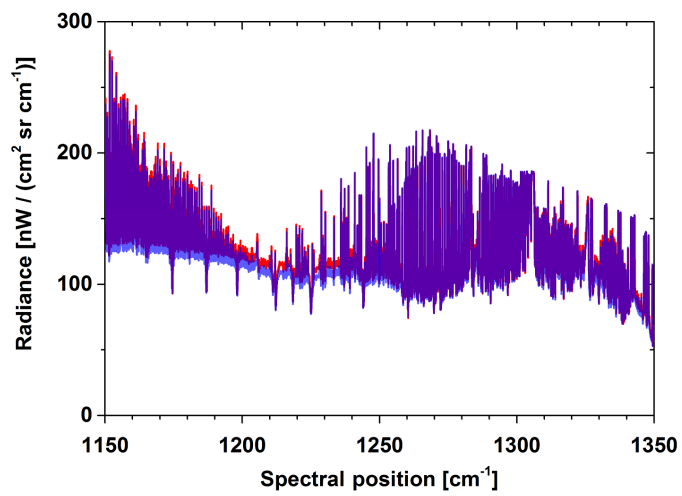

Figure B2. Sensitivity simulations investigating the effect of decreasing mode radii on the simulated spectral signatures of $\beta$-NAT particles for a T-Matrix scenario with $\mathrm{AR}=0.1$ (blue) and a corresponding Mie scenario $(\mathrm{AR}=1.0$, red). (a, b) Large particle mode, size distribution B1. (c, d) Intermediate particle mode, size distribution B1a. (e, f) Small particle mode, size distribution B1b. Numbers above panels (a), (c), and (e) indicate corresponding mode radii and particle number densities (see Fig. B1). "TM" corresponds to T-matrix simulation and "SP" to spheroid (numbers indicate AR). 
Acknowledgements. The authors thank Myasishchev Design Bureau and the ESSenCe coordination team for a successful Geophysica field campaign. ESSenCe was supported by the European Space Agency/Mission Science Division under the ESSenCe project (Technical Assistance for the Deployment of Airborne Limbsounders during ESSenCe). W. Woiwode is grateful to the Karlsruhe House for Young Scientists for supporting a 5-month research stay at NASA Langley airborne Research Center (NASA LaRC, Hampton, USA), and thanks M. C. Pitts and L. R. Poole from the CALIPSO PSC team for a great and productive time at NASA LaRC. We thank M. I. Mishchenko (NASA Goddard Institute for Space Studies, New York, USA) for helpful recommendations and providing the contact with $\mathrm{L}$. Bi (Department of Atmospheric Sciences, Texas A\&M University, College Station, USA, now at School of Earth Sciences, Zhejiang University, Hangzhou, China), who performed the extensive IIM + SOV T-Matrix calculations. For calculations for moderately aspherical particles, we used the double-precision T-Matrix code for randomly oriented nonspherical particles provided by M. I. Mishchenko, L. D. Travis, and D. W. Mackowski at http://www.giss.nasa.gov/staff/mmishchenko/t_matrix.html. The data set for the simulation of highly aspherical $\beta$-NAT particles used here is available from L. Bi (bilei@zju.edu.cn). We thank U. M. Biermann, L. J. Richwine, R. F. Niedziela, and A. Y. Zasetsky for providing the refractive indices of $\beta$-NAT, $\alpha$-NAT, STS, NAD, and ice. The CLaMS simulation was performed using computing time granted on the supercomputer JUROPA at Jülich Supercomputing Centre (JSC) under VSR project ID JICG11. We thank EMCWF for the data used for the MIPAS-STR retrievals, the CLaMS simulation, and the potential vorticity map. We thank Wyoming Atmospheric Soundings (Department of Atmospheric Science, University of Wyoming, USA) for providing the radiosonde data (see http://weather.uwyo.edu/upperair/sounding.html). We acknowledge the Physical Sciences Division, Earth System Research Laboratory, NOAA, Boulder, Colorado, USA, for providing the sea surface temperature data (see http://www.esrl.noaa.gov/psd/). We thank R. Müller (Institute of Energy and Climate Research (IEK-7), Forschungszentrum Jülich GmbH, Germany) for helpful comments. We thank two anonymous referees, H. Grothe, and M. J. Rossi for helpful comments. We acknowledge support by the Deutsche Forschungsgemeinschaft and the Open Access Publishing Fund of the Karlsruhe Institute of Technology.

The article processing charges for this open-access

publication were covered by a Research

Centre of the Helmholtz Association.

Edited by: H. Saathoff

Reviewed by: two anonymous referees

\section{References}

Bi, L., Yang, P., Kattawar, G. W., and Mishchenko, M. I.: Efficient implementation of the invariant imbedding T-matrix method and the separation of variables method applied to large nonspherical inhomogeneous particles, J. Quant. Spectrosc. Ra., 116, 169$183,2013$.
Biermann, U. M.: Gefrier- und FTIR-Experimente zur Nukleation und Lebensdauer stratosphärischer Wolken, $\mathrm{PhD}$ thesis, Universität Bielefeld, cuvillier Verlag, ISBN 3-89712-212-X, 1998.

Biermann, U. M., Crowley, J. N., Huthwelker, T., Moortgat, G. K., Crutzen, P. J., and Peter, T.: FTIR studies on lifetime prolongation of stratospheric ice particles due to NAT coating, Geophys. Res. Lett., 25, 3939-3942, doi:10.1029/1998GL900040, 1998.

Biermann, U. M., Luo, B. P., and Peter, T.: Absorption spectra and optical constants of binary and ternary solutions of $\mathrm{H}_{2} \mathrm{SO}_{4}, \mathrm{HNO}_{3}$, and $\mathrm{H}_{2} \mathrm{O}$ in the mid infrared at atmospheric temperatures, J. Phys. Chem. A, 104, 783-793, 2000.

Borrmann, S., Luo, B., and Mishchenko, M.: The application of the T-matrix method to the measurement of aspherical particles with forward scattering optical particle counters, J. Aerosol Sci., 31, 789-799, 2000.

Brooks, S. D., Baumgardner, D., Gandrud, B., Dye, J. E., Northway, M. J., Fahey, D. W., Bui, T. P., Toon, O. B., and Tolbert, M. A.: Measurements of large stratospheric particles in the Arctic polar vortex, J. Geophys. Res., 108, 4652, doi:10.1029/2002JD003278, 2003.

CALIPSO Science Team: CALIPSO/CALIOP Level 2, Polar Stratospheric Cloud Data, version 1.00, Hampton, VA, USA: NASA Atmospheric Science Data Center (ASDC), doi:10.5067/CALIOP/CALIPSO/CAL_LID_L2_PSCMask-

Prov-V1-00_L2-001.00 (last access: 6 October 2014), 2015.

Carslaw, K. S., Kettleborough, J. A., Northway, M. J., Davies, S., Gao, R., Fahey, D. W., Baumgardner, D. G., Chipperfield, M. P., and Kleinböhl, A.: A vortex-scale simulation of the growth and sedimentation of large nitric acid hydrate particles, J. Geophys. Res., 107, 8300, doi:10.1029/2001JD000467, 2002.

Drdla, K., Gandrud, B. W., Baumgardner, D., Wilson, J. C., Bui, T. P., Hurst, D., Schauffler, S. M., Jost, H., Greenblatt, J. B., and Webster, C. R.: Evidence for the widespread presence of liquidphase particles during the 1999-2000 Arctic winter, J. Geophys. Res., 108, 8318, doi:10.1029/2001JD001127, 2003.

Fahey, D. W., Gao, R. S., Carslaw, K. S., Kettleborough, J., Popp, P. J., Northway, M. J., Holecek, J. C., Ciciora, S. C., McLaughlin, R. J., Thompson, T. L., Winkler, R. H., Baumgardner, D. G., Gandrud, B., Wennberg, P. O., Dhaniyala, S., McKinley, K., Peter, T., Salawitch, R. J., Bui, T. P., Elkins, J. W., Webster, C. R., Atlas, E. L., Jost, H.,Wilson, J. C., Herman, R. L., Kleinböhl, A., and von König, M.: The detection of large $\mathrm{HNO}_{3}$-containing particles in the winter Arctic stratosphere, Science, 291, 1026-1031, 2001.

Fischer, H., Birk, M., Blom, C., Carli, B., Carlotti, M., von Clarmann, T., Delbouille, L., Dudhia, A., Ehhalt, D., Endemann, M., Flaud, J. M., Gessner, R., Kleinert, A., Koopman, R., Langen, J., López-Puertas, M., Mosner, P., Nett, H., Oelhaf, H., Perron, G., Remedios, J., Ridolfi, M., Stiller, G., and Zander, R.: MIPAS: an instrument for atmospheric and climate research, Atmos. Chem. Phys., 8, 2151-2188, doi:10.5194/acp-8-2151-2008, 2008.

Grooß, J.-U., Engel, I., Borrmann, S., Frey, W., Günther, G., Hoyle, C. R., Kivi, R., Luo, B. P., Molleker, S., Peter, T., Pitts, M. C., Schlager, H., Stiller, G., Vömel, H., Walker, K. A., and Müller, R.: Nitric acid trihydrate nucleation and denitrification in the Arctic stratosphere, Atmos. Chem. Phys., 14, 1055-1073, doi:10.5194/acp-14-1055-2014, 2014.

Grothe, H., Myhre, C. E. L., and Tizek, H., Vibrational spectra of nitric acid dihydrate (NAD), Vib. Spectrosc., 34, 55-62, 2004. 
Grothe, H., Tizek, H., Waller, D., and Stokes, D. J.: The crystallization kinetics and morphology of nitric acid trihydrate, Phys. Chem. Chem. Phys., 8, 2232-2239, doi:10.1039/B601514J, 2006.

Hanson, D. and Mauersberger, K.: Laboratory studies of the nitric acid trihydrate: implications for the south polar stratosphere, Geophys. Res. Lett., 15, 855-858, doi:10.1029/GL015i008p00855, 1988.

Höpfner, M.: Study on the impact of polar stratospheric clouds on high resolution mid-IR limb emission spectra, J. Quant. Spectrosc. Ra., 83, 93-107, 2004.

Höpfner, M. and Emde, C.: Comparison of single and multiple scattering approaches for the simulation of limb-emission observations in the mid-IR, J. Quant. Spectrosc. Ra., 91, 275-285, 2004.

Höpfner, M., Oelhaf, H., Wetzel, G., Friedl-Vallon, F., Kleinert, A., Lengel, A., Maucher, G., Nordmeyer, H., Glatthor, N., Stiller, G. P., von Clarmann, T., Fischer, H., Kröger, C., and Deshler, T.: Evidence of scattering of tropospheric radiation by PSCs in mid-IR limb emission spectra: MIPAS-B observations and KOPRA simulations, Geophys. Res. Lett., 29, 119-1-119-4, doi:10.1029/2001GL014443, 2002.

Höpfner, M., Luo, B. P., Massoli, P., Cairo, F., Spang, R., Snels, M., Di Donfrancesco, G., Stiller, G., von Clarmann, T., Fischer, H., and Biermann, U.: Spectroscopic evidence for NAT, STS, and ice in MIPAS infrared limb emission measurements of polar stratospheric clouds, Atmos. Chem. Phys., 6, 1201-1219, doi:10.5194/acp-6-1201-2006, 2006a.

Höpfner, M., Larsen, N., Spang, R., Luo, B. P., Ma, J., Svendsen, S. H., Eckermann, S. D., Knudsen, B., Massoli, P., Cairo, F., Stiller, G., v. Clarmann, T., and Fischer, H.: MIPAS detects Antarctic stratospheric belt of NAT PSCs caused by mountain waves, Atmos. Chem. Phys., 6, 1221-1230, doi:10.5194/acp-6-1221-2006, 2006b.

Hoyle, C. R., Engel, I., Luo, B. P., Pitts, M. C., Poole, L. R., Grooß, J.-U., and Peter, T.: Heterogeneous formation of polar stratospheric clouds - Part 1: Nucleation of nitric acid trihydrate (NAT), Atmos. Chem. Phys., 13, 9577-9595, doi:10.5194/acp13-9577-2013, 2013.

Iannarelli, R. and Rossi, M. J.: Heterogeneous Kinetics of $\mathrm{H}_{2} \mathrm{O}, \mathrm{HNO}_{3}$ and $\mathrm{HCl}$ on $\mathrm{HNO}_{3}$ hydrates $(\alpha$-NAT, $\beta$-NAT, NAD) in the range 175-200 K, Atmos. Chem. Phys. Discuss., doi:10.5194/acp-2016-247, in review, 2016.

Kaufmann, M., Blank, J., Friedl-Vallon, F., Gerber, D., Guggenmoser, T., Höpfner, M., Kleinert, A., Sha, M. K., Oelhaf, H., Riese, M., Suminska-Ebersoldt, O., Woiwode, W., Siddans, R., Kerridge, B., Moyna, B., Rea, S., and Oldfield, M.: Technical Assistance for the Deployment of Airborne Limbsounders during ESSenCe, Tech. rep., European Space Agency, available at: https://earth.esa.int/documents/ 10174/134665/ESSenCe_Final_Report (last access: 28 July 2016), 2013.

Kaye, P., Hirst, E., Greenaway, R., Ulanowski, Z., Hesse, E., DeMott, P., Saunders, C., and Connolly, P.: Classifying atmospheric ice crystals by spatial light scattering, Opt. Lett., 33, 1545-1547, doi:10.1364/OL.33.001545, 2008.

Kim, Y., Choi, W., Lee, K., Park, J. H., Massie, S. T., Sasano, Y., Nakajima, H., and Yokota, T.: Polar stratospheric clouds observed by the ILAS-II in the Antarctic region: Dual compositions and variation of compositions during June to August of 2003, J. Geophys. Res., 111, D13S90, doi:10.1029/2005JD006445, 2006.

Lambert, A., Santee, M. L., Wu, D. L., and Chae, J. H.: A-train CALIOP and MLS observations of early winter Antarctic polar stratospheric clouds and nitric acid in 2008, Atmos. Chem. Phys., 12, 2899-2931, doi:10.5194/acp-12-2899-2012, 2012.

Libbrecht, K. G.: The physics of snow crystals, Rep. Prog. Phys., 68, 855-895, 2005.

Meyer, J., Rolf, C., Schiller, C., Rohs, S., Spelten, N., Afchine, A., Zöger, M., Sitnikov, N., Thornberry, T. D., Rollins, A. W., Bozóki, Z., Tátrai, D., Ebert, V., Kühnreich, B., Mackrodt, P., Möhler, O., Saathoff, H., Rosenlof, K. H., and Krämer, M.: Two decades of water vapor measurements with the FISH fluorescence hygrometer: a review, Atmos. Chem. Phys., 15, 85218538, doi:10.5194/acp-15-8521-2015, 2015.

Mishchenko, M. I. and Travis, L. D.: Capabilities and limitations of a current FORTRAN implementation of the T-matrix method for randomly oriented rotationally symmetric scatterers, J. Quant. Spectrosc. Ra., 60, 309-324, 1998.

Molleker, S., Borrmann, S., Schlager, H., Luo, B., Frey, W., Klingebiel, M., Weigel, R., Ebert, M., Mitev, V., Matthey, R., Woiwode, W., Oelhaf, H., Dörnbrack, A., Stratmann, G., Grooß, J.U., Günther, G., Vogel, B., Müller, R., Krämer, M., Meyer, J., and Cairo, F.: Microphysical properties of synoptic-scale polar stratospheric clouds: in situ measurements of unexpectedly large $\mathrm{HNO}_{3}$-containing particles in the Arctic vortex, Atmos. Chem. Phys., 14, 10785-10801, doi:10.5194/acp-14-10785-2014, 2014

Murphy, D. M. and Koop, T.: Review of the vapour pressures of ice and supercooled water for atmospheric applications, Q. J. Roy. Meteor. Soc., 131, 1539-1565, 2005.

Nash, E. R., Newman, P. A., Rosenfield, J. E., and Schoeberl, M. R.: An objective determination of the polar vortex using Ertel's potential vorticity, J. Geophys. Res., 101, 9471-9478, 1996.

Newman, S. M., Smith, J. A., Glew, M. D., Rogers, S. M., and Taylor, J. P.: Temperature and salinity dependence of sea surface emissivity in the thermal infrared, Q. J. Roy. Meteorol. Soc., 131, 2539-2557, 2005.

Niedziela, R. F., Miller, R. E., and Worsnop, D. R.: Temperature and frequency-dependent optical constants for nitric acid dehydrate from aerosol spectroscopy, J. Phys. Chem. A, 102, 6477-6484, 1998.

Ortega, I. K., Maté, B., Moreno, M. A., Herrero, V. J., and Escribano, R.: Infrared spectra of nitric acid trihydrate $(\beta$-NAT): A comparison of available optical constants and implication for the detection of polar stratospheric clouds (PSCs), Geophys. Res. Lett., 33, L19816, doi:10.1029/2006GL026988, 2006.

Peter, T.: Microphysics and Heterogeneous Chemistry of Polar Stratospheric Clouds, Annu. Rev. Phys. Chem., 48, 785-822, doi:10.1146/annurev.physchem.48.1.785, 1997.

Piesch, C., Gulde, T., Sartorius, C., Friedl-Vallon, F., Seefeldner, M., Wölfel, M., Blom, C. E., and Fischer, H.: Design of a MIPAS instrument for high-altitude aircraft, Proc. of the 2nd Internat. Airborne Remote Sensing Conference and Exhibition, ERIM, Ann Arbor, MI, Vol. II, San Francisco, California, 24-27 June 1996, 199-208, 1996.

Pitts, M. C., Poole, L. R., Dörnbrack, A., and Thomason, L. W.: The 2009-2010 Arctic polar stratospheric cloud season: a CALIPSO perspective, Atmos. Chem. Phys., 11, 2161-2177, doi:10.5194/acp-11-2161-2011, 2011. 
Pitts, M. C., Poole, L. R., Lambert, A., and Thomason, L. W.: An assessment of CALIOP polar stratospheric cloud composition classification, Atmos. Chem. Phys., 13, 2975-2988, doi:10.5194/acp-13-2975-2013, 2013.

Remedios, J. J., Leigh, R. J., Waterfall, A. M., Moore, D. P., Sembhi, H., Parkes, I., Greenhough, J., Chipperfield, M. P., and Hauglustaine, D.: MIPAS reference atmospheres and comparisons to V4.61/V4.62 MIPAS level 2 geophysical data sets, Atmos. Chem. Phys. Discuss., 7, 9973-10017, doi:10.5194/acpd-79973-2007, 2007.

Richwine, L. J., Clapp, M. L., Miller, R. E., and Worsnop, D. R.: Complex refractive indices in the infrared of nitric acid trihydrate aerosols, Geophys. Res. Lett., 22, 2625-2628, 1995.

Spang, R. and Remedios, J. J.: Observations of a distinctive infrared spectral feature in the atmospheric spectra of polar stratospheric clouds measured by the CRISTA instrument, Geophys. Res. Lett., 30, 1875, doi:10.1029/2003GL017231, 2003.

Spang, R., Remedios, J. J., and Barkley, M. P.: Colour indices for the detection and differentiation of cloud types in infra-red limb emission spectra, Adv. Space Res., 33, 1041-1047, 2004.

Spang, R., Remedios, J. J., Kramer, L. J., Poole, L. R., Fromm, M. D., Müller, M., Baumgarten, G., and Konopka, P.: Polar stratospheric cloud observations by MIPAS on ENVISAT: detection method, validation and analysis of the northern hemisphere winter 2002/2003, Atmos. Chem. Phys., 5, 679-692, doi:10.5194/acp-5-679-2005, 2005.

Stefanutti, L., Sokolov, L., Balestri, S., MacKenzie, A. R., and Khattatov, V.: The M-55 Geophysica as a platform for the airborne polar experiment, J. Atmos. Ocean. Tech., 16, 1303-1312, doi:10.1175/1520-0426(1999)016<1303:tmgaap>2.0.co;2, 1999.

Stetzer, O., Möhler, O., Wagner, R., Benz, S., Saathoff, H., Bunz, H., and Indris, O.: Homogeneous nucleation rates of nitric acid dihydrate (NAD) at simulated stratospheric conditions Part I: Experimental results, Atmos. Chem. Phys., 6, 3023-3033, doi:10.5194/acp-6-3023-2006, 2006.

Stiller, G. P., von Clarmann, T., Funke, B., Glatthor, N., Hase, F., Höpfner, M., and Linden, A.: Sensitivity of trace gas abundances retrievals from infrared limb emission spectra to simplifying approximations in radiative transfer modelling, J. Quant. Spectrosc. Ra., 72, 249-280, doi:10.1016/S0022-4073(01)00123-6, 2002.

Tizek, H., Knözinger, E., and Grothe, H.: Formation and phase distribution of nitric acid hydrates in the mole fraction range $x_{\text {HNO3 }}<0.25$ : A combined XRD and IR study, Phys. Chem. Chem. Phys., 6, 972-979, doi:10.1039/B310672A, 2004.

Toon, O. B., Tolbert, M. A., Koehler, B. G., Middlebrook, A. M., and Jordan, J.: Infrared optical constants of $\mathrm{H}_{2} \mathrm{O}$ ice, amorphous nitric acid solutions, and nitric acid hydrates, J. Geophys. Res., 99, 25631-25654, doi:10.1029/94JD02388, 1994.

Voigt, C., Schreiner, J., Kohlmann, A., Zink, P., Mauersberger, K., Larsen, N., Deshler, T., Kröger, C., Rosen, J., Adriani, A., Cairo, F., Di Donfrancesco, G., Viterbini, M., Ovarlez, J., Ovarlez, H., David, C., and Dörnbrack, A.: Nitric Acid Trihydrate (NAT) in Polar Stratospheric Clouds, Science, 290, 1756-1758, 2000. von Clarmann, T., Höpfner, M., Kellmann, S., Linden, A., Chauhan, S., Funke, B., Grabowski, U., Glatthor, N., Kiefer, M., Schieferdecker, T., Stiller, G. P., and Versick, S.: Retrieval of temperature, $\mathrm{H}_{2} \mathrm{O}, \mathrm{O}_{3}, \mathrm{HNO}_{3}, \mathrm{CH}_{4}, \mathrm{~N}_{2} \mathrm{O}, \mathrm{ClONO}_{2}$ and $\mathrm{ClO}$ from MIPAS reduced resolution nominal mode limb emission measurements, Atmos. Meas. Tech., 2, 159-175, doi:10.5194/amt-2-159-2009, 2009.

Waibel, A. E., Peter, Th., Carslaw, K. S., Oelhaf, H., Wetzel, G., Crutzen, P. J., Pöschl, U., Reimer, E., and Fischer, H.: Arctic ozone loss due to denitrification, Science, 283, 2064-2069, 1999.

Waterman, P.: Symmetry, unitarity, and geometry in electromagnetic scattering, Phys. Rev., 3, 825-839, 1971.

Weiss, F., Kubel, F., Gálvez, O., Hoelzel, M., Parker, S. F., Iannarelli, R., Rossi, M. J., and Grothe, H.: Metastable Nitric Acid Trihydrate in Ice Clouds, Angew. Chem. Int. Edit., 55, 3276-3280, doi:10.1002/anie.201510841, 2016.

Woiwode, W., Oelhaf, H., Gulde, T., Piesch, C., Maucher, G., Ebersoldt, A., Keim, C., Höpfner, M., Khaykin, S., Ravegnani, F., Ulanovsky, A. E., Volk, C. M., Hösen, E., Dörnbrack, A., Ungermann, J., Kalicinsky, C., and Orphal, J.: MIPAS-STR measurements in the Arctic UTLS in winter/spring 2010: instrument characterization, retrieval and validation, Atmos. Meas. Tech., 5, 1205-1228, doi:10.5194/amt-5-1205-2012, 2012.

Woiwode, W., Grooß, J.-U., Oelhaf, H., Molleker, S., Borrmann, S., Ebersoldt, A., Frey, W., Gulde, T., Khaykin, S., Maucher, G., Piesch, C., and Orphal, J.: Denitrification by large NAT particles: the impact of reduced settling velocities and hints on particle characteristics, Atmos. Chem. Phys., 14, 11525-11544, doi:10.5194/acp-14-11525-2014, 2014.

Woiwode, W., Suminska-Ebersoldt, O., Oelhaf, H., Höpfner, M., Belyaev, G. V., Ebersoldt, A., Friedl-Vallon, F., Grooß, J.-U., Gulde, T., Kaufmann, M., Kleinert, A., Krämer, M., Kretschmer, E., Kulessa, T., Maucher, G., Neubert, T., Piesch, C., Preusse, P., Riese, M., Rongen, H., Sartorius, C., Schardt, G., Schönfeld, A., Schuettemeyer, D., Sha, M. K., Stroh, F., Ungermann, J., Volk, C. M., and Orphal, J.: Validation of first chemistry mode retrieval results from the new limb-imaging FTS GLORIA with correlative MIPAS-STR observations, Atmos. Meas. Tech., 8, 2509-2520, doi:10.5194/amt-8-2509-2015, 2015.

Worsnop, D. R., Zahniser, M. S., Fox, L. E., and Wofsy, S. C.: Vapor Pressures of Solid Hydrates of Nitric Acid: implications for Polar Stratospheric Clouds, Science, 259, 71-74, 1993.

Zasetsky, A. Y., Khalizov, A. F., Earle, M. E., and Sloan, J. J.: Frequency Dependent Complex Refractive Indices of Supercooled Liquid Water and Ice Determined from Aerosol Extinction Spectra, J. Phys. Chem. A, 109, 2760-2764, 2005.

Zöger, M., Afchine, A., Eicke, N., Gerhards, M. T., Klein, E., McKenna, D. S., Morschel, U., Schmidt, U., Tan, V., Tuitjer, F., Woyke, T., and Schiller, C.: Fast in situ stratospheric hygrometers: a new family of balloon-borne and airborne Lyman alpha photofragment fluorescence hygrometers, J. Geophys. Res., 104, 1807-1816, 1999. 